\title{
The Genome-Wide Binding Profile for Human RE1 Silencing Transcription Factor Unveils a Unique Genetic Circuitry in Hippocampus
}

\author{
James C. McGann, ${ }^{1,2 *}$ Michael A. Spinner, ${ }^{1 *}$ Saurabh K. Garg, ${ }^{1,3 *}$ Karin A. Mullendorff,, ${ }^{1,4}$ Randall L. Woltjer, ${ }^{5}$ \\ and Gail Mandel ${ }^{1}$ \\ ${ }^{1}$ Vollum Institute, Oregon Health and Science University, Portland, Oregon 97239, ${ }^{2}$ Cancer Early Detection Advanced Research Center, Oregon Health and \\ Science Institute, Portland, Oregon 97239, ${ }^{3}$ Department of Cutaneous Oncology, H. Lee Moffitt Cancer Center and Research Institute, Tampa, Florida 33612, \\ ${ }^{4}$ Universidad San Sebastian, Puerto Montt, Chile 5501842, and ${ }^{5}$ Department of Pathology, Division of Neuropathology, Oregon Health and Science \\ University, Portland, Oregon 97239
}

Early studies in mouse neurodevelopment led to the discovery of the RE1 Silencing Transcription Factor (REST) and its role as a master repressor of neuronal gene expression. Recently, REST was reported to also repress neuronal genes in the human adult brain. These genes were found to be involved in pro-apoptotic pathways; and their repression, associated with increased REST levels during aging, were found to be neuroprotective and conserved across species. However, direct genome-wide REST binding profiles for REST in adult brain have not been identified for any species. Here, we apply this approach to mouse and human hippocampus. We find an expansion of REST binding sites in the human hippocampus that are lacking in both mouse hippocampus and other human non-neuronal cell types. The unique human REST binding sites are associated with genes involved in innate immunity processes and inflammation signaling which, on the basis of histology and recent public transcriptomic analyses, suggest that these new target genes are repressed in glia. We propose that the increases in REST expression in mid-adulthood presage the beginning of brain aging, and that human REST function has evolved to protect the longevity and function of both neurons and glia in human brain.

Key words: Alzheimer's disease; ChIP-seq; hippocampus; innate immunity; NRSF; REST

\section{Significance Statement}

The RE1 Silencing Transcription Factor (REST) repressor has served historically as a model for gene regulation during mouse neurogenesis. Recent studies of REST have also suggested a conserved role for REST repressor function across lower species during aging. However, direct genome-wide studies for REST have been lacking for human brain. Here, we perform the first genome-wide analysis of REST binding in both human and mouse hippocampus. The majority of REST-occupied genes in human hippocampus are distinct from those in mouse. Further, the REST-associated genes unique to human hippocampus represent a new set related to innate immunity and inflammation, where their gene dysregulation has been implicated in aging-related neuropathology, such as Alzheimer's disease.

\section{Introduction}

The role of transcription factors during the development and early maturation of the nervous system has been the subject of intense research, as new sequencing technologies have been

Received Aug. 5, 2020; revised May 12, 2021; accepted June 16, 2021

Author contributions: J.C.M., M.A.S., S.K.G., and G.M. designed research; J.C.M., M.A.S., S.K.G., K.A.M., and R.L.W. performed research; J.C.M., M.A.S., S.K.G., R.L.W., and G.M. contributed unpublished reagents/analytic tools; J.C.M., M.A.S., S.K.G., K.A.M., R.L.W., and G.M. analyzed data; J.C.M. wrote the first draft of the paper; J.C.M., M.A.S., S.K.G., K.A.M., R.L.W., and G.M. edited the paper; G.M. wrote the paper.

This work was supported by National Institutes of Health Grant R01 NS099374 to G.M. We thank all members of the G.M. laboratory for discussion and support; staff at the Oregon Health and Science University MPSSR for expertise; and Drs. Guanming Wu and Andrew Adey (Oregon Health and Science University) for critical comments on the statistical and bioinformatics analyses. brought to bear on neurodevelopmental disorders, such as autism, and aging disorders, such as Alzheimer's disease (AD). A factor of interest in this regard is the RE1 Silencing Transcription Factor (REST, Chong et al., 1995; also called

All mouse procedures were approved by the Institutional Animal Care and Use Committee at Oregon Health and Science University (No. IP00000284) and performed in accordance with approved guidelines. ChIPseq datasets generated in the current study have been deposited in the NCBI Gene Expression Omnibus database (https://www.ncbi.nlm.nih.gov/geo/) and are accessible through accession number GSE144226. Previously published datasets used for comparative analysis are listed and cited in Extended Data Fig. 4-1.

*J.C.M., M.A.S., and S.K.G. contributed equally to this work.

The authors declare no competing financial interests.

Correspondence should be addressed to Gail Mandel at mandelg@ohsu.edu.

https://doi.org/10.1523/JNEUROSCI.2059-20.2021

Copyright $\odot 2021$ the authors 
NRSF, Schoenherr and Anderson, 1995). During embryogenesis, REST is expressed primarily in non-neural tissues in the embryo (D. Zhang et al., 2017), as well as in embryonic stem cells (ESCs) and embryonic and adult neuronal progenitors (Mukherjee et al., 2016). In non-neuronal cell types and ESCs, REST binds to thousands of target genes, both coding and noncoding, containing its consensus binding site, the 21-nucleotide Repressor Element 1 (RE1, Kraner et al., 1992; NRSE, Schoenherr et al., 1996; D. S. Johnson et al., 2007; Otto et al., 2007). The relief of REST-mediated repression, including dismissal of its corepressors during differentiation (Ballas et al., 2005), endows mature phenotypic features on neurons, including expression of voltagesensitive and ligand-gated ion channels, growth factors, and their cognate receptors, synaptic proteins, and neuronal transcription factors (Ballas and Mandel, 2005).

Historically, most work on REST has focused exclusively on its important role in forming the mouse embryonic nervous system. More recent studies, however, indicate that REST also plays a role later in life. For example, several studies in mice indicate that REST is present in certain populations of postmitotic neurons in late embryogenesis, persisting in the postnatal brain (Palm et al., 1998; Calderone et al., 2003; Kuwabara et al., 2004; Sun et al., 2005; Gao et al., 2011; Nechiporuk et al., 2016). REST levels are also increased under conditions of ischemia or seizure in adult mouse brain, and preventing the increase alleviates neuronal cell death associated with both conditions (Calderone et al., 2003; Hu et al., 2011; Noh et al., 2012; Kaneko et al., 2014; McClelland et al., 2014; Singh-Taylor et al., 2018). Two studies have pointed to a protective function for increased REST expression in human neurons during aging through binding to stressrelated and pro-apoptotic genes (Lu et al., 2014; Zullo et al., 2019). Further, REST levels were reduced in the age-related cognitive disease, $\mathrm{AD}$ ( $\mathrm{Lu}$ et al., 2014). In the same study, loss of REST in mouse resulted in increased cortical activity, and loss of function of a REST ortholog in Caenorhabditis elegans resulted in increased neural activity and an increase in lifespan (Zullo et al., 2019).

Several molecular and bioinformatics findings, however, question the extent to which mice model REST function in human brain overall. First, while REST initially appeared with the evolution of a vertebrate nervous system (Mortazavi et al., 2006), REST has also evolved with strong positive selection in humans, specifically (Aigner and Yeo, 2009; Mozzi et al., 2017). Second, comparisons of REST binding profiles in human and mouse ESCs, and across other human cell types, indicate an expansion of the human cistrome, suggesting unique roles for REST-regulated genes in human brain development and neurodevelopmental disease (R. Johnson et al., 2009; Rockowitz et al., 2014; Rockowitz and Zheng, 2015; D. Wang et al., 2018). Third, while the cognate REST binding site (RE1) sequence is highly conserved across species (Mortazavi et al., 2006), many RE1 sequences are unique to the human genome ( $\mathrm{R}$. Johnson et al., 2009; Rockowitz and Zheng, 2015). These findings notwithstanding, direct studies of REST binding sites have not been determined by genome-wide REST binding profiles in the human brain.

Here, we address this question using chromatin immunoprecipitation with massively parallel DNA sequencing (ChIP-seq) on mouse and human hippocampus. We find little overlap between REST binding sites in these two species. Further, previous immunohistochemistry and recent public transcriptomic data indicate that the predominant origin of our REST binding sites is likely glia, highlighting a role for non-neuronal cells in protecting the longevity of neural cells.

\section{Materials and Methods}

\section{Mouse strains}

REST $^{\mathbf{G T}(\boldsymbol{D O A} \text { TE11) }}$ (Rest ${ }^{G T}$ ) mutants were established by blastocyst injection of the D047E11 GT clone (GenBank Acc No. DU821609) (Hansen et al., 2003). REST ${ }^{G T i(D 047 E 11)}$ mice carrying the inverted GT vector $\left(R e s t^{G T i}\right.$ ) were obtained by crossing to Flpe deleter mice (Rodríguez et al., 2000). This colony is maintained and genotyped to generate brain-specific $R_{E S T}{ }^{-1-}$ mice using Nestin Cre as described by Nechiporuk et al. (2016). Mice were backcrossed to WT C57Bl6 line (Jackson ImmunoResearch Laboratories, strain 000664) for at least 10 generations.

\section{Human tissue}

Tissue was procured through the Oregon Alzheimer's Disease Center and Oregon Health and Science University Department of Pathology and subjects had no significant brain pathologies, except age-related changes as established by review of clinical histories and survey of common neurodegenerative disease-associated lesions. Tissues were deidentified, and their use was exempted from review by the Institutional Review Board at Oregon Health and Science University in accordance with relevant guidelines. Informed consent was obtained from all participants and/or their legal representatives. All analyses were performed on postmortem frozen tissue with a time interval from death to preservation of $<24 \mathrm{~h}$

\section{Immunohistochemistry}

Human tissues were evaluated as described previously (Nguyen et al., 2018). In brief, brains were fixed in neutral-buffered formaldehyde solution for at least 2 weeks and examined grossly and microscopically. For microscopic evaluation, tissue samples were processed into paraffin blocks and $6 \mu \mathrm{m}$ sections were stained with H\&E and Luxol fast blue and immunostained as described above after antigen retrieval with development using $\mathrm{ABC}$ kits from Vector Laboratories.

\section{Antibodies}

Mouse anti-actin (DSHB clone JLA20-C, RRID:AB_528068, WB-1:5000, Fig. $1 C, D$ ), mouse anti- $\alpha$-tubulin (DSHB clone AA4.3, RRID:AB 579793, WB-1:5000; Fig. 2B), and rabbit anti-HDAC2 (Invitrogen, RRID:AB_2547079, WB-1:5000; Fig. 2A); Rabbit anti-human REST antibody REST-C (Ballas et al., 2005) (in-house, WB-1:1000, Figs. 1 and 2; and all ChIP experiments).

\section{Western blotting of human and mouse hippocampi}

Tissue removal: Human hippocampi were dissected on dry ice and flash frozen using precooled steel plates at $-70^{\circ} \mathrm{C}$ within $24 \mathrm{~h}$ of removal from human postmortem brain and stored at $-90^{\circ} \mathrm{C}$. Mouse hippocampi were flash frozen in liquid nitrogen before dissection on dry ice. For the blots: Human hippocampal tissue was lysed in cold lysis buffer containing $50 \mathrm{~mm}$ Tris, pH 7.5, $150 \mathrm{~mm} \mathrm{NaCl}, 1 \mathrm{~mm}$ EDTA, $0.1 \mathrm{~mm}$ sodium vanadate, $10 \mathrm{~mm} \beta$-glycerophosphate, $10 \mathrm{~mm} \mathrm{NaF}$, protease inhibitors (Roche Basel), 1\% Triton X-100, and 10\% glycerol and resolved on $3 \%-8 \%$ precast gradient Tris-acetate gels (Invitrogen). For mouse tissue, nuclear extracts were prepared by the modified Dignam method as previously described (Grimes et al., 2000). For both human and mouse, probing the blots with primary antibodies was conducted at $4^{\circ} \mathrm{C}$ overnight followed by incubation with the appropriate secondary antibodies for $2 \mathrm{~h}$ at room temperature. REST signals were visualized using the chemiluminescent West Pico detection kit (Thermo Fisher Scientific, catalog \#34083). Western blot quantification was conducted in ImageJ (Schindelin et al., 2012) and performed by subtracting the background signal (region below individual bands) from the REST signal. REST signal was normalized to loading controls recognized by either HDAC2 or $\alpha$-tubulin. 


\section{REST immunoprecipitation after cell transfection}

Confluent HEK-293 cells were transfected in 6-well plates with Lipofectamine 2000 (Thermo Fisher Scientific, catalog \#11668027) using $2 \mu \mathrm{g}$ of either human or mouse full-length cDNA in pcDNA3.1 expression vectors under control of the CMV promoter (Thermo Fisher Scientific, catalog \#V79520) and empty vector control. Cells were lysed with ChIP buffer $(10 \mathrm{~mm}$ Tris-HCl, pH 8.0, 1\% Triton X-100, 150 mm $\mathrm{NaCl}$, and $1 \mathrm{~mm}$ EDTA, and protease inhibitors) in a Dounce homogenizer on ice. Lysate protein concentrations were determined by detergent compatible protein assay (Bio-Rad, catalog \#5000112). For the immunoprecipitations, Protein G Dynabeads (Invitrogen, catalog \#10003D) were preequilibrated in TBSTBp (TBS with $1 \%$ BSA, $1 \%$ Triton $\mathrm{X}-100$, and protease inhibitors). Lysates were first precleared by mixing up to $20 \mu \mathrm{g}$ of lysate with $10 \mu \mathrm{l}$ of equilibrated Protein $\mathrm{G}$ beads in ChIP buffer for $4 \mathrm{~h}$ at $4^{\circ}$ C. For antibody binding, $20 \mu \mathrm{l}$ of Protein $\mathrm{G}$ beads were incubated with $\leq 20 \mu$ g (Fig. $1 B$ ) of anti-REST-C antibodies (Ballas et al., 2005) for $2 \mathrm{~h}$ at room temperature in $0.5 \mathrm{ml}$ TBSTBp. The bead-Ab complex was washed 3 times with TBSTBp, and then added to the precleared lysate and incubated overnight at $4^{\circ} \mathrm{C}$. Beads were washed three times with ChIP buffer at $4^{\circ} \mathrm{C}$ for $5 \mathrm{~min}$ and protein was eluted with $20 \mu \mathrm{l}$ of $100 \mathrm{~mm}$ glycine, $\mathrm{pH} 2.6$, at room temperature. Western blotting to quantify the amount of REST in the immunoprecipitates was done by loading equal volumes of the immunoprecipitated eluates, as well as $20 \mu \mathrm{g}$ of the unprecipitated lysate (input, maximum possible REST signal among trials). The blots were processed for REST as above for the tissues and visualized using a LICOR Odyssey CLx imager. REST signals were quantified in ImageJ as above, and reported as \% of REST signal relative to input. All gels were imaged, processed, and quantified using identical settings.

\section{REST ChIP}

Approximately 100-200 mg of unfixed frozen tissue from pooled mouse hippocampi (ages 5 weeks) or individual human hippocampus (ages 63, 63, and 67 years) was cross-linked in cross-linking buffer (10 mм HEPES, pH 7.2, $100 \mathrm{~mm} \mathrm{NaCl}, 1 \mathrm{~mm}$ EDTA, and 1 mM EGTA) containing $1 \%$ formaldehyde (Thermo Fisher Scientific; methanol free) for $10 \mathrm{~min}$ at room temperature. Excess formaldehyde was quenched with $125 \mathrm{~mm}$ glycine for $5 \mathrm{~min}$ at room temperature. Tissue was resuspended in homogenization buffer ( $250 \mathrm{~mm}$ sucrose, $25 \mathrm{~mm} \mathrm{KCl}, 5 \mathrm{mM} \mathrm{MgCl}_{2}, 20 \mathrm{~mm}$ tricine-KOH; pH 7.8, 1 mм DTT, $0.15 \mathrm{~mm}$ spermine, $0.5 \mathrm{~mm}$ spermidine, and protease inhibitors) after a wash with ice-cold PBS. Tissue was homogenized using Dounce homogenizer with loose (A) and tight (B) pestle ( 5 strokes each), followed by additional 5 strokes of tight pestle with $0.3 \% \mathrm{NP}-40$, and then the homogenate was passed through a $40 \mu \mathrm{m}$ strainer. Nuclei were isolated at 4000 $\times g$ at $4^{\circ} \mathrm{C}$ for $5 \mathrm{~min}$ and resuspended in $\sim 0.5 \mathrm{ml}$ sonication buffer (10 mм Tris-HCl, pH 8.0, 1 mм EDTA, 1 mм EGTA). Fragmented Student's $t$ test).
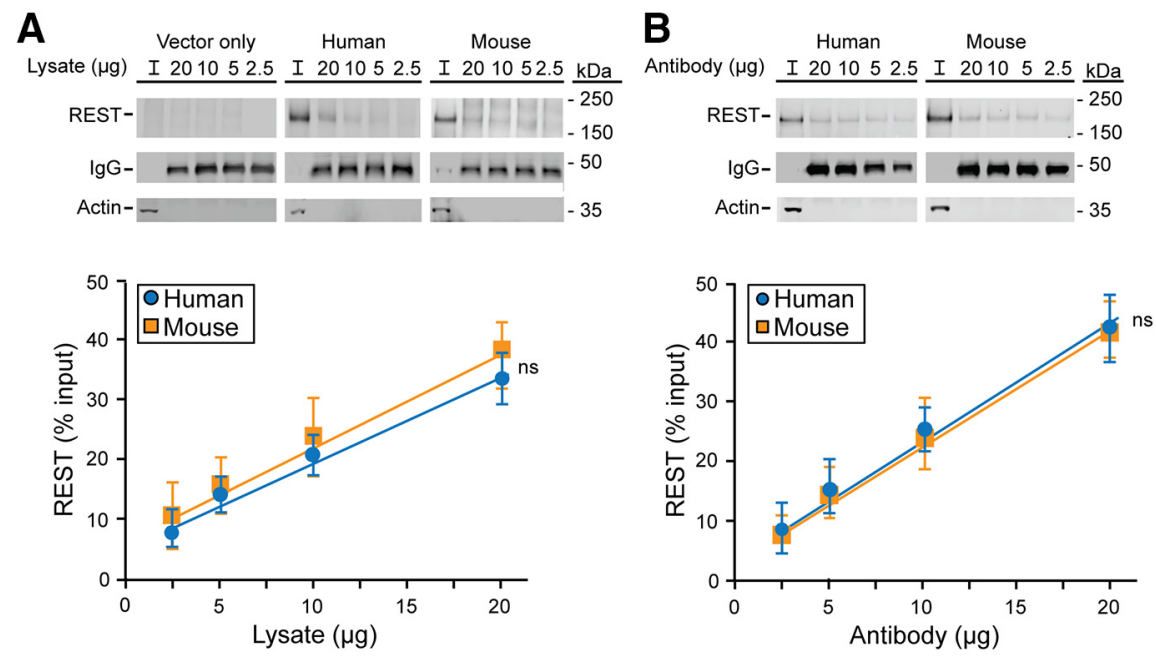

Figure 1. The REST-C antibody immunoprecipitates mouse and human REST protein expressed in human embryonic kidney cells with equal efficiency. $\boldsymbol{A}, \boldsymbol{B}$, Top, Representative Western blots of REST in immunoprecipitates from human embryonic kidney cells transfected with equal molar amounts of indicated vector CDNAs. REST migrates at $\sim 200 \mathrm{kDa}$. I, Input. REST level in $20 \mu \mathrm{g}$ of lysate representing the maximal signal for REST. IgG heavy chain signal serves as control for equal amounts antibodybead complex during the immunoprecipitation, except in $\boldsymbol{B}$, where antibody amount was intentionally reduced across samples. Actin was used to normalize the input concentration for the independent transfections. Bottom, Quantification of REST in the Western blots. Graphs represent the averages of four independent transfections. $y$ axis, amount of REST in the immunoprecipitates relative to the amount of REST in the starting lysate (Input). Error bars indicate SEM. ns, Not significant $(p=0.93$,

A

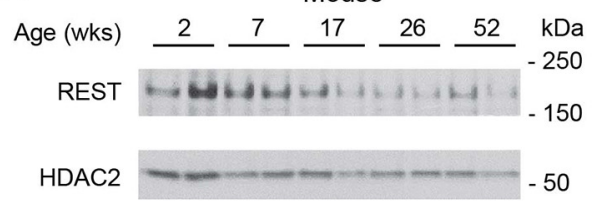

Figure 2. REST protein levels increase with advanced age in human but not mouse hippocampus. $\boldsymbol{A}, \boldsymbol{B}$, Top, Representative Western blots of mouse and human hippocampal extracts probed with the REST-C antibody. REST migrates at $\sim 200 \mathrm{kDa}$. $\alpha$-Tubulin and HDAC2, loading control. Bottom, Quantification of Western blots for REST from mouse and human. Each symbol represents an individual mouse $(n=20)$ or human donor $(n=24)$. In human samples, REST levels show a correlation with age $(R=0.74, p=3.87 \mathrm{E}-05)$. chromatin was prepared from $\sim 10$ million nuclei in Covaris S220 (200 cycle per burst, $5 \%$ duty cycle at power level 4 for $12 \mathrm{~min}$ at $4^{\circ}$ C) in presence of $0.3 \%$ SDS. For the immunprecipitations, Protein G Dynabeads (Invitrogen; $30 \mu \mathrm{l} /$ reaction) were equilibrated in TBSTBp (TBS with $1 \%$ BSA, $1 \%$ Triton X-100, and protease inhibitors); $10 \mu \mathrm{l}$ Protein $\mathrm{G}$ beads were used for preclearing the chromatin in ChIP buffer (10 mm Tris- $\mathrm{HCl}, \mathrm{pH} 8.0,1 \%$ Triton X-100, 150 $\mathrm{mm} \mathrm{NaCl}$, and $1 \mathrm{~mm}$ EDTA, and protease inhibitors) for $4 \mathrm{~h}$ at $4^{\circ} \mathrm{C}$. $20 \mu \mathrm{l}$ Protein $\mathrm{G}$ beads were used to make the bead-Ab complex with $20 \mu \mathrm{g}$ anti-REST-C antibodies (Ballas et al., 2005) for $2 \mathrm{~h}$ at room temperature in $0.5 \mathrm{ml}$ TBSTBp. After washing bead-Ab complex 3 times with TBSTBp, ChIP was conducted overnight at $4^{\circ} \mathrm{C}$ 
with precleared chromatin. Beads were washed twice with low-salt, high-salt, and $\mathrm{LiCl}$ buffers at $4^{\circ} \mathrm{C}$, followed by two washes with $\mathrm{TE}$ at room temperature. DNA was eluted and crosslinks were reversed overnight at $65^{\circ} \mathrm{C}$. Contaminants were removed from DNA by RNaseA and Proteinase K treatment followed by purification with PCR purification kit (QIAGEN).

\section{REST ChIP-seq}

Ten nanograms of fragmented DNA was used as input for a modified TruSeq Nano DNA library preparation protocol (Illumina). Briefly, input DNA was treated with $3^{\prime}$ to $5^{\prime}$ exonuclease activity and $5^{\prime}$ to $3^{\prime}$ polymerase activity to blunt the ends. There was no size selection of the fragments. A single "A" nucleotide was added to the 3 ' ends to enhance ligation to the adapters. RNA adapters (Illumina) were ligated to the fragmented DNA followed by cleanup using sample purification beads provided with the kit. The ligation product was enriched using 14 cycles of PCR. The amplification product was cleaned using sample purification beads. Libraries were profiled using the TapeStation D1000 DNA tape. Library concentrations were determined using the Library Quantification Kit for Illumina sequencing platforms (Kapa Biosystems) on a StepOnePlus Real Time PCR Workstation (Thermo Fisher Scientific). Libraries were mixed for multiplexing, and the concentration of the mix was determined by real-time PCR. Short read sequencing was performed on a HiSeq 2500 (Illumina) using a single 100 cycle protocol by the Oregon Health and Science University Massively Parallel Sequencing Shared Resource. Base call files were converted to fastq files using Bcl2Fastq2 (Illumina).

\section{ChIP-seq analysis}

Sequenced reads were processed and analyzed by the ENCODE ChIPseq analysis pipeline (https://github.com/ENCODE-DCC/chip-seqpipeline2) using the GRCh38 (hg38) version of the human genome and the mm10 version of the mouse genome. Three human replicates (age 63,63 , and 67 years) were processed. For mice, two replicates of pooled hippocampi from 4 animals were analyzed. Peaks for each replicate were called, and then the final peak list was based on the optimal set calculated using the SPP peak caller and the irreproducible discovery rate (IDR), which incorporates all the replicates into the analysis.

\section{Read quality analysis, gene annotation, and Gene Ontology (GO) enrichment}

REST peaks for each experiment were converted into bed files and analyzed using the R programs Homer (Heinz et al., 2010), ChIPseeker (Yu et al., 2015), ClusterProfiler (Yu et al., 2012), and EnrichR (Chen et al., 2013). REST target genes associated with all peaks used for GO analysis were annotated with the seq2gene function in ChIPseeker. REST target genes were defined as genes with REST peaks located within the gene body and including up to $5 \mathrm{~kb}$ upstream of the Refseq-annotated transcriptional start site (TSS). "Core" REST peaks were defined as peaks that overlapped in at least 40 datasets of the 48 non-neuronal REST ChIP-seq replicates publicly available from ENCODE as determined by Homer (Extended Data Fig. 4-1). GO lists were reduced in complexity by the PANTHER "slim" database (Mi et al., 2019), and overrepresentation and enrichment of GO terms were calculated using Fisher's exact test with Bonferroni correction. Motif analysis was performed using DREME (Bailey, 2011) and DIVERSITY (Mitra et al., 2018) on all REST peaks from mouse or human hippocampus.

\section{High Occupancy Target (HOT) region filtering}

As known HOT regions predominately center around highly expressed TSSs (Chen et al., 2014), we used aggregate plots for ChIP-seq read depths for $\mathrm{H} 3 \mathrm{~K} 4 \mathrm{me} 3$ and $\mathrm{H} 3 \mathrm{~K} 9 \mathrm{ac}$ modifications to simulate HOT regions in hippocampal tissue and the cell lines analyzed. We then calculated an active chromatin score for each putative HOT region and compared them with REST enrichment levels. We first tested our filter method on two independent ENCODE datasets from human ESCs and the human A459 lung carcinoma cell line (Extended Data Fig. 4-1), and were able to establish a threshold that eliminated the majority of the TSS $\mathrm{H} 3 \mathrm{~K} 4 \mathrm{me} 3$ and $\mathrm{H} 3 \mathrm{~K} 9 \mathrm{ac}$ peaks, $63 \%$ and $79 \%$, respectively, while maintaining the majority, $87 \%$ and $99 \%$, of REST peaks containing consensus RE1 sites (Fig. 4C). We then extended the filter to ENCODE ChIP-seq analysis on human $\mathrm{H} 1$ neurons and our human hippocampal dataset, normalizing the thresholds to median REST and H3K4me3 and H3K9ac ChIP-seq reads for each dataset. The filter significantly reduced the proportion of HOT regions with minimal loss of REST peaks in all of these cell types and human hippocampus and, importantly, in hippocampal peaks associated with REST RE1 sequences (Fig. 4D,E). As predicted, the filtered peaks centered on promoter regions, and the closest annotated genes to the filtered peaks were enriched significantly for housekeeping functions atypical of REST target genes and typical of HOT regions, such as ribosome biogenesis, splicing, and DNA metabolism. In total, our filter removed 11,495 of 13,620 REST peaks.

\section{Results}

\section{Divergent postnatal REST expression patterns in mouse and human hippocampus support distinct REST functions in humans}

For the ChIP-seq analysis, we used an affinity-purified polyclonal antibody validated previously in our laboratory for ChIP-seq analyses (Nechiporuk et al., 2016). The antibody was generated against the conserved C-terminus of human REST (65\% identical and $75 \%$ similar at the amino acid level between mouse and human) and was validated for REST specificity in both Western blotting and qChIP analyses of mouse tissue using a REST gene trap KO line that prevents expression of the full-length transcript (Nechiporuk et al., 2016). Three previously published studies (Ballas et al., 2005; McGann et al., 2014; Nechiporuk et al., 2016), in culture and in vivo, indicate that the same REST antibody used for this ChIP-seq analysis detects REST expression over a wide range of endogenous REST levels, from very high (mouse ESCs), to intermediate (neural progenitors), to minimal or undetectable levels in neurons. To our knowledge, mESCs express the highest level of REST in any cell type or tissue.

To test directly for equal efficiency of the REST antibody to immunoprecipitate human and mouse REST, we transfected human or mouse cDNA into human embryonic kidney cells that express negligible levels of endogenous REST. We then immunoprecipitated REST over an eightfold range of lysate concentrations from each transfection. Conversely, we used an eightfold range of REST antibody to immunoprecipitate a fixed concentration of lysate. REST protein in the immunoprecipitates was quantitated by Western blotting. We found equivalent immunoprecipitation efficiencies for the two species in both experiments (new Fig. 1A,B). Of note, this REST antibody will not detect truncated splice variants that are still largely enigmatic in terms of their function (Chen and Miller, 2018).

Consistent with the idea that REST function could be different in mouse and human, a comparative Western blotting experiment indicated a completely opposite time course for REST expression, with advanced age, in mouse and human hippocampus. Mouse REST levels dropped dramatically, to a barely detectable level by 4-6 months of age that persisted well into adulthood (18 months; Fig. 2A). In contrast, human REST levels increased progressively with age as reported previously (Lu et al., 2014) (Fig. 2B). We were not expecting the dramatic decrease in mouse REST to minimal levels, which for technical reasons restricted the age for an in-depth ChIP-seq analysis. However, at 5 weeks of age, which we chose for the analysis, the hippocampal neural cells are mature and the mice exhibit adult behaviors (Semple et al., 2013). The questions of the mechanisms regulating the differential REST postnatal expression profiles during aging in the two species, and whether the cellular distribution of 
A

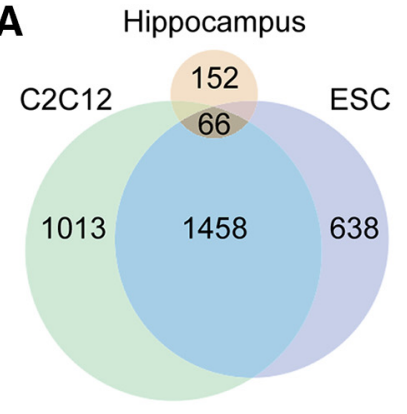

C

Consensus RE1 sequence $(n=49)$

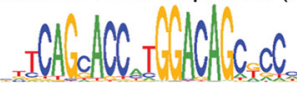

Left half-site $(n=16)$

CCACCACCCAGACCATGTCC

* Insertion $(n=15)$

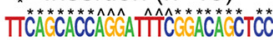

Right half-site $(n=7)$

CGÄGGGTCCCFTCAACACACCCCCC

Degenerate $(n=15)$

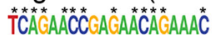

B

Hipp.

ESC

C2C12

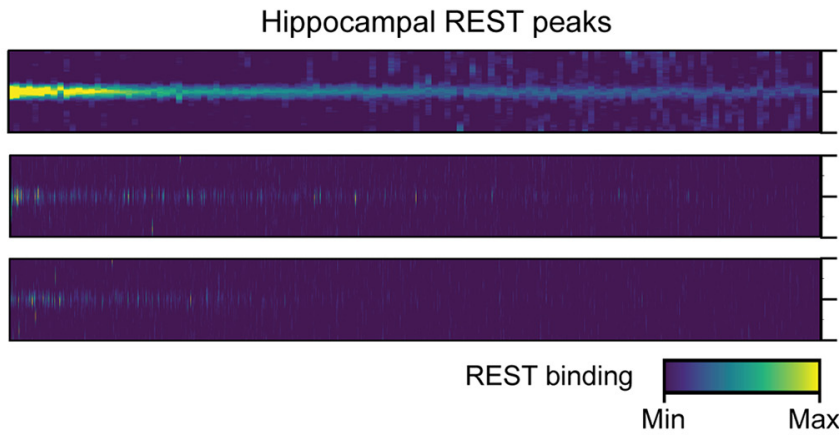

D

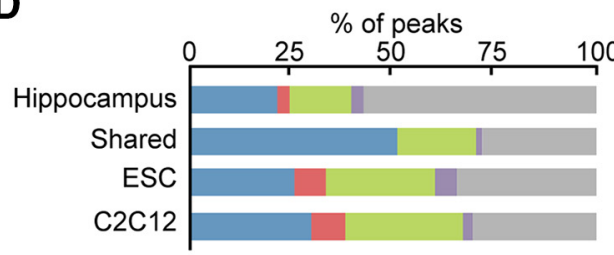

Nearest Gene Annotation

Promoter

Exon

Intron

Downstream

Distal Intergenic

E

$\mathbf{F}$
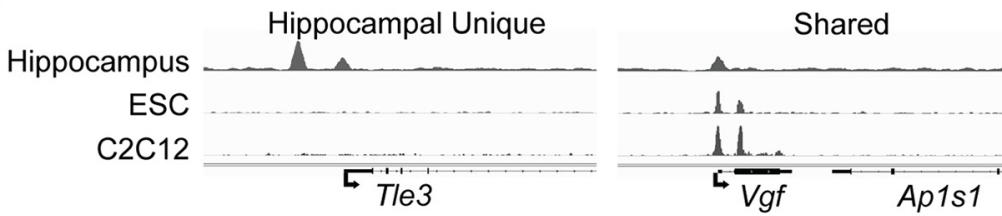

$\mathrm{ESC}+\mathrm{C} 2 \mathrm{C} 12$
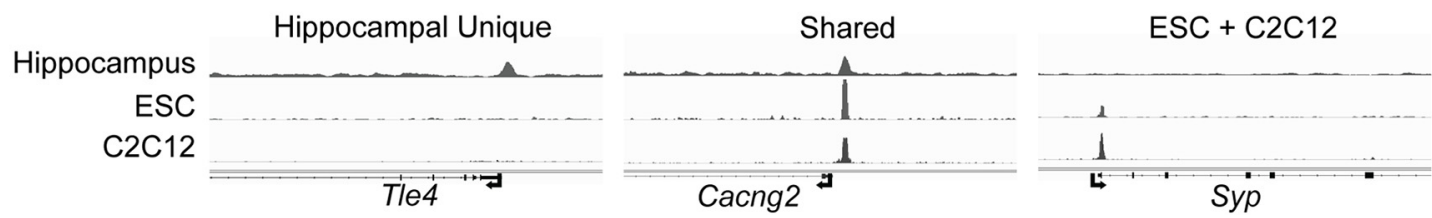

Figure 3. REST ChIP-seq analysis reveals associated genes encoding neurodevelopmental proteins unique to mouse hippocampus. $\boldsymbol{A}$, Venn diagram of REST peaks in mouse hippocampus and indicated cell types. (2C12, mouse myoblast cell line. A full list of mouse hippocampal peaks and their locations can be found in Extended Data Figure 3-1. B, Heat maps of REST binding density at ChIP-seq peaks. Each heat map is scaled to the maximum level of REST bound in each tissue/cell type, and regions are sorted from highest to lowest REST binding levels in hippocampus (Hipp) and indicated cell lines. C, Consensus and related RE1 sequences (Mortazavi et al., 2006; Johnson et al., 2007; 0tto et al., 2007). Values in parentheses indicate the number of hippocampal REST ChIP-seq peaks associated with given motif. *Nucleotides matching RE1 consensus. ^Inserted nucleotides in consensus. D, Distribution of REST peaks at RE1 consensus and related sequences relative to the nearest annotated gene. $\boldsymbol{E}, \mathrm{GO}$ categories for genes associated with REST peaks unique to the hippocampus. Negative log 10 of $p$ value was calculated by Fisher's exact test and corrected by FDR (see Materials and Methods). Dashed line indicates $p=0.05$. A full list of G0 categories can be found in Extended Data Figure 3-2. F, REST peaks at representative genomic loci. Tle loci are unique to hippocampus, and shared loci indicated are prototypical neuronal function RE1 sequences. All REST peaks shown have underlying RE1 or related sequences with the exception of Tle3. Arrows indicate TSSs. Broader horizontal lines in genes indicate exons.

REST expression is altered with age in each species, are large questions in themselves. Here we focus specifically on the first comparative genomic REST binding analysis in the two species at one adult stage.

REST-bound sites in the mouse postnatal hippocampus As a first step in our ChIP-seq analysis, we compared the RESTbound peaks in mouse hippocampus with REST peaks found previously in ESCs (Nechiporuk et al., 2016) and the C2C12 muscle cell line (Fig. 3A) (McGann et al., 2014; Davis et al., 2018). We identified only 221 REST-bound peaks in the mouse hippocampus (Extended Data Fig. 3-1). We found that 69\% of the peaks were unique to hippocampus, with the remainder shared with $\mathrm{C} 2 \mathrm{C} 12$ and ESCs that had 2537 and 2162 REST peaks, respectively (Fig. $3 A, B$ ). The lower number of hippocampal REST peaks likely reflects a lower REST expression level in 
mouse hippocampus. This conclusion is supported by the ability of this antibody to bind to exogenous REST epitopes over a large dynamic range (Fig. 1), as well as by previous studies of endogenous REST where this same antibody detected epitopes from mESCs, which express very high levels of REST, to fully differentiated neurons that express extremely low levels of REST (Ballas et al., 2005; He et al., 2020).

We sought to determine whether the unique REST-bound hippocampal peaks were associated with genes lacking consensus and related RE1 sequences typical of non-neuronal cells and neural progenitors, because binding to non-RE1 sequences has been reported previously (Rockowitz and Zheng, 2015; D. Wang et al., 2018). For this analysis, we used consensus RE1 motifs, RE1 half motifs, and other RE1-related motifs (Fig. 3C) (Mortazavi et al., 2006; D. S. Johnson et al., 2007; Otto et al., 2007). As expected, all hippocampal peaks shared with non-neuronal cells displayed consensus or RE1-related sequences (Table 1). However, of the REST peaks unique to the hippocampus, none overlaid a consensus RE1 sequence and only $22 \%$ were associated with an RE1-related sequence (Table 1; Extended Data Fig. 3-1). In contrast, consensus and RE1-related peaks represented $88 \%$ and $82 \%$, respectively, of the sites unique to each nonneuronal cell type (Table 1 ). The distribution of REST peaks shared among all three tissue/cell samples, which contained RE1 sequences, was biased more toward the promoter regions of annotated genes (Fig. 3D), consistent with previous studies in mouse ESCs (McGann et al., 2014; Rockowitz and Zheng, 2015). The distribution of REST peaks unique to hippocampus was biased away from promoters and more toward intergenic regions. More than $50 \%$ of the hippocampalunique peaks belonged to this category, compared with the peaks shared among all cell types (only $\sim 25 \%$ intergenic peaks) (Fig. 3D). This finding is commensurate with a previous observation that nonconserved sites tend to be located more distally from promoters (R. Johnson et al., 2009).

Most REST binding sites in mice have been associated with canonical neuronal genes that encode proteins related to synaptic or neuronal functions. To determine the gene classes bound by REST in our study, we first defined REST target genes as having REST peaks in the gene body, extending from $5 \mathrm{~kb}$ upstream of the Refseq-annotated TSS to $3 \mathrm{~kb}$ downstream of the 3' untranslated regions. For the REST peaks shared among hippocampus and the non-neuronal cell types, all of which contained RE1 or RE1-related sequences, the GO enrichments were very similar to those from either ESCs or C2C12 cells (Extended Data Fig. 3-2) and neural progenitors (Ballas et al., 2005). These GO categories represented prototypical REST-regulated proteins important in neurodevelopment, such as glutamatergic synaptic transmission (33-fold enrichment, GO:0035249), neurotransmitter binding (14-fold enrichment, GO:0042165), and postsynaptic membrane localization (GO:0045211). In contrast to these shared neuronal REST target genes, supported by many experimental studies, GO terms for the target genes associated with unique hippocampal REST peaks showed enrichments for less stereotypical functions (Fig. 3E,F), including enrichment categories for $\mathrm{Wnt}$ proteins and transcription factors. The latter category was represented exclusively by several paralogs of the transducing-like enhancer of split family of transcriptional co-repressors (Tle1, Tle3, Tle4, and Tle6; Fig. 3E). These transcription factors are known to be important regulators of differentiation in stem/progenitor cells, both in early neurogenesis and in adult stem/progenitor cell populations where REST is present (Roth et al., 2010; J. Wang et al., 2010; Mukherjee et al., 2016).
Table 1. REST peak number and underlying RE1 motif status for mouse hippocampus, mESCs, and $\mathrm{mC2C} 12$ myoblasts $^{a}$

\begin{tabular}{|c|c|c|c|}
\hline Tissue/cell type & $\begin{array}{l}\text { With consensus } \\
\text { RE1 }\end{array}$ & $\begin{array}{l}\text { With related } \\
\text { RE1 }\end{array}$ & $\begin{array}{l}\text { Lack any } \\
\text { RE1 }\end{array}$ \\
\hline Hippocampal unique (152) & 0 & 33 & 119 \\
\hline Hippocampal, ESC, C2C12, shared (66) & 48 & 18 & 0 \\
\hline ESC, C2C12, shared (1458) & 1105 & 351 & 2 \\
\hline ESC unique (638) & 292 & 271 & 75 \\
\hline C2C12 unique (1013) & 331 & 497 & 185 \\
\hline
\end{tabular}

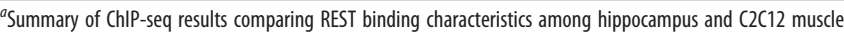
cells and ESCs. $m$ refers to mouse. Numbers in parentheses indicate total peak numbers.

We also noted the existence of many REST peaks coinciding with RE1 sequences in ESCs and C2C12 cells, where REST binding was not detected in the hippocampus (Fig. $3 A, D$; Extended Data Figs. 3-1 and 3-2). This result supports prior studies indicating that REST binding is partially cell typespecific (Rockowitz et al., 2014).

\section{REST-bound sites in human hippocampus}

For our genome-wide REST binding analysis of human hippocampus, given that the increase in REST levels is a continuum with age, we selected $\sim 60$-year-old postmortem samples as an intermediate expression level between the ages of 40 and 100 (Fig. 2B). Unexpectedly, on our initial investigation of the REST binding profiles, we found that a preponderance of the REST peaks coincided with genes that were highly expressed and encoded ubiquitously expressed proteins, contrary to the stereotypical function of REST target genes. Indeed, these REST peaks coincided with HOT regions, or phantom or hyper-ChIPable peaks, that have been identified in previous ChIP-seq analyses for other transcription factors across species (Park et al., 2013; Teytelman et al., 2013; Krebs et al., 2014; Jain et al., 2015; Wreczycka et al., 2019). HOT regions are considered transcription factor binding artifacts as they are in the 99th percentile of genomic regions in the ENCODE database based on their cumulative density of ChIP-seq enrichments (Wreczycka et al., 2019). Further, HOT regions tend to be proximal to promoters driving high expression of housekeeping genes across many cell types (Wreczycka et al., 2019), as we found for our REST peaks. In addition to their presence within a potential HOT region, many REST peaks fulfilled these other criteria: (1) present in the HOT regions independent of the ENCODE cell type or REST antibody used for the ChIP analyses, including pulldown of a GFP-REST fusion protein with GFP antibody (Fig. 4A,B; ENCODE lines used in Extended Data Fig. 4-1), (2) present in a HOT region from a control ChIP-seq using IgG for the pulldown (Fig. 4A), and (3) present in HOT regions located proximal to the TSSs, as defined by ChIP-seq analyses for RNA polymerase II and chromatin markers of active gene expression (Fig. 4A).

Authentic genomic targets of a specific transcription factor were identified previously by filtering out hyper-ChIPable peaks using a cell or animal model deleted for the transcription factor (Krebs et al., 2014). Because our analysis used postmortem brain tissue, we used a bioinformatics filter for our data (see Materials and Methods). In brief, we used the aggregate plots for ChIP-seq read depths for $\mathrm{H} 3 \mathrm{~K} 4 \mathrm{me} 3$ and $\mathrm{H} 3 \mathrm{~K} 9 \mathrm{ac}$ modifications as a proxy for the HOT regions in the ENCODE dataset and compared these read depths to the level of REST enrichment at hippocampal sites (bigwigAverageOverBed; Fig. 4C). We then set a threshold that removed the majority of known HOT regions while maintaining REST peaks with RE1 and RE1-related sites (Fig. 4D,E). Our 
A
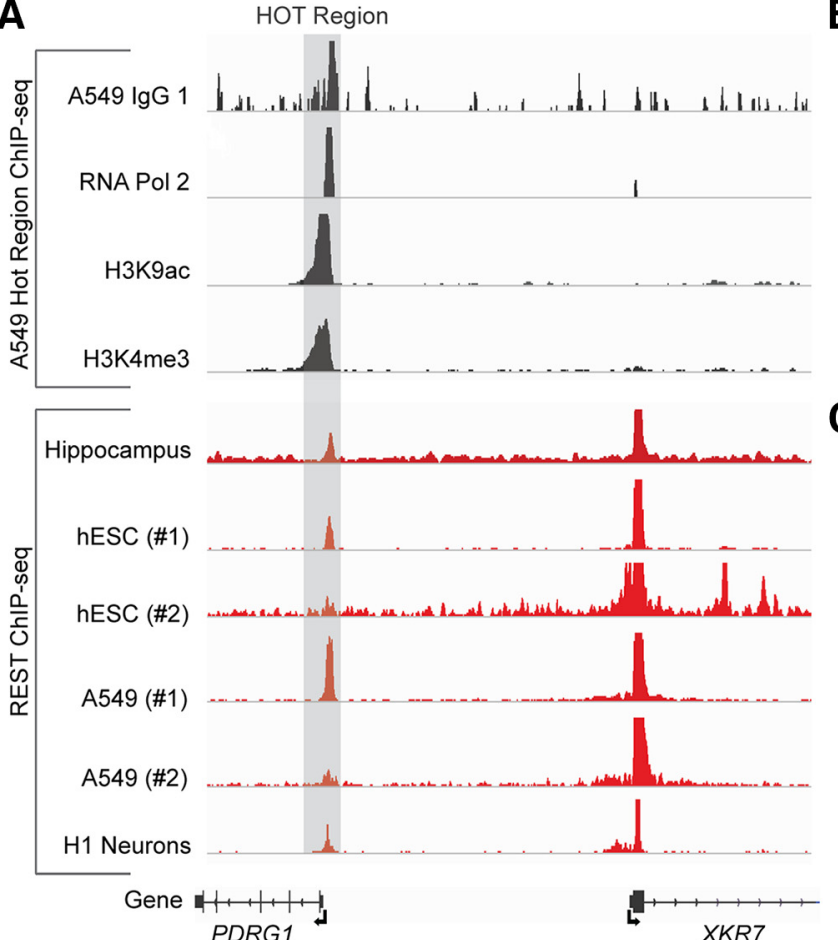

PDRG1

D

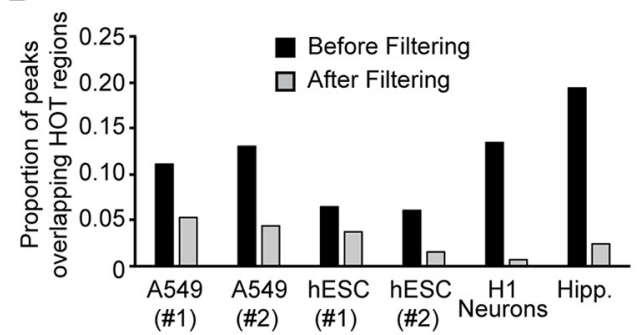

B

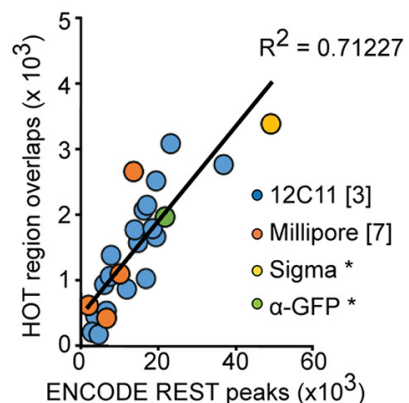

C
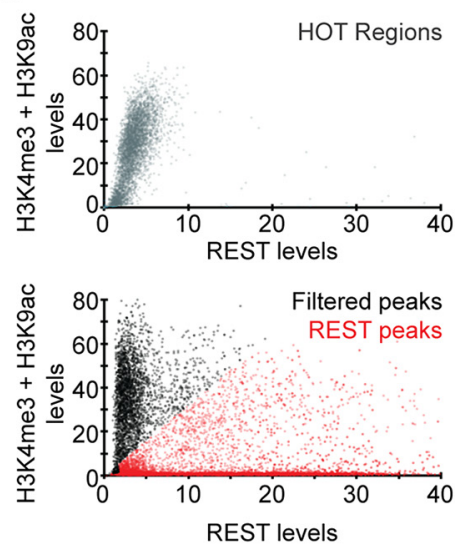

E

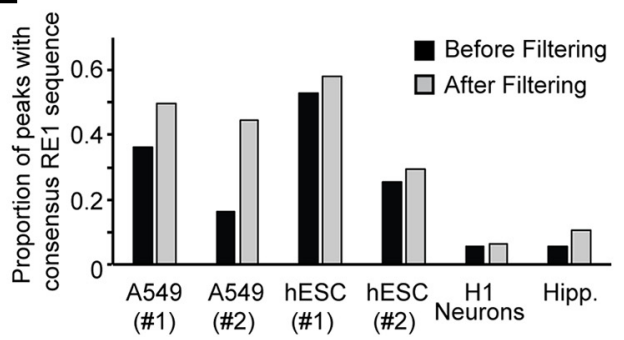

Figure 4. Bioinformatic filtering of human hippocampal REST ChIP-seq peaks diminishes HOT regions while preserving a majority of REST peaks. All ChIP-seq data other than this study are from ENCODE or indicated references. A, Top, ChlP-seq tracks in A549 cells showing HOT regions in two genes relative to TSSs as defined by ChlP-seq analyses for RNA polymerase 2 and active transcription markers (H3K9Ac; H3K4me3) as proxy for HOT regions. Note the HOT region even in the lgG1 ChlP-seq control track (ENCFF593HFQ). Bottom, Our REST ChIP-seq peaks in human hippocampus at the same genomic regions as in A549 cells (top), relative to ENCODE REST peaks in the indicated cell types. $\boldsymbol{B}$, Scatter plot of REST ChIP-seq peaks using different antibody sources and their correlation with annotated HOT regions ( $\max =4320)$ (Wreczycka et al., 2019). *Source of antibodies used found in Extended Data Figure 4-1. C, Scatter plots of REST ChIP-seq data from the A549 cells at annotated HOT regions (top) or all REST peaks (bottom), color-coded for peaks that pass the filter (red) or those that are filtered out from further analysis (black). $\boldsymbol{D}, \boldsymbol{E}$, Histograms of the proportion REST ChIP-seq peaks that remain after filtering in indicated cell lines and human H1 neurons. $\boldsymbol{D}$, Proportion of total REST peaks that overlap HOT regions. $\boldsymbol{E}$, Proportion of REST peaks with underlying consensus RE1 sequences.

subsequent analyses of hippocampal REST ChIP-seq data included only the REST peaks that remained after applying the filter.

REST-bound peaks in human hippocampus show limited overlap with REST peaks in non-neuronal cell types and are not associated with prototypical REST-repressed genes We identified a total of 2125 hippocampal REST peaks after filtering (Fig. 5A; Extended Data Fig. 5-1). Of these, 89\% were unique relative to previously annotated REST peaks, defined as RESTbound peaks in $>40$ of 48 ENCODE replicates from human nonneuronal cell lines ("Core" peaks; Fig. 5A; see Materials and Methods), consistent with a previous study indicating that REST occupancy is cell-specific (Bruce et al., 2004). Further, heat maps of REST ChIP densities showed that the full set of hippocampal peaks had distinct signatures among the publicly available REST ChIP-seq datasets (Fig. 5B). The unique hippocampal REST peaks were associated with a much higher percentage of peaks lacking underlying consensus and related RE1 sequences compared with the Core REST peaks (38\% vs 5\%, respectively; Table 2). The genomic loci shared between hippocampus and Core were both biased more toward promoter regions (Fig. 5C). Predictably, the genes associated with the shared peaks, exemplified in HeLa cells and hESCs, were enriched for the typical neuronal GO categories, including growth factors, ion channels, neurotransmitter receptors, and synaptic adhesion molecules (Fig. 5D; Extended Data Fig. 5-1). We noted that REST enrichment in the hippocampal unique peaks was overall lower than REST enrichment in the peaks shared with the Core dataset (Fig. 5E). However, lower levels of REST enrichment were also observed for cell-specific peaks in ESCs and A549 cells when each was compared with the other cell types in the Core dataset (Fig. 5E), and is consistent with a previous study indicating that more ancient (i.e., conserved RE1 sites) have higher affinities than lineage-specific sites (R. Johnson et al., 2009). 
A

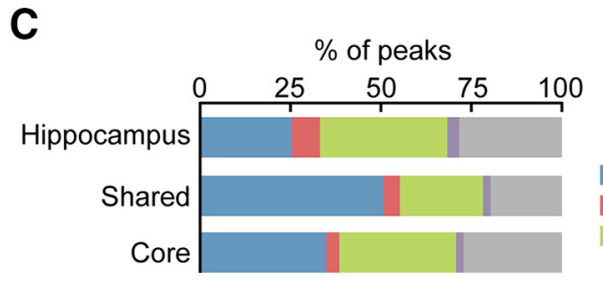

B

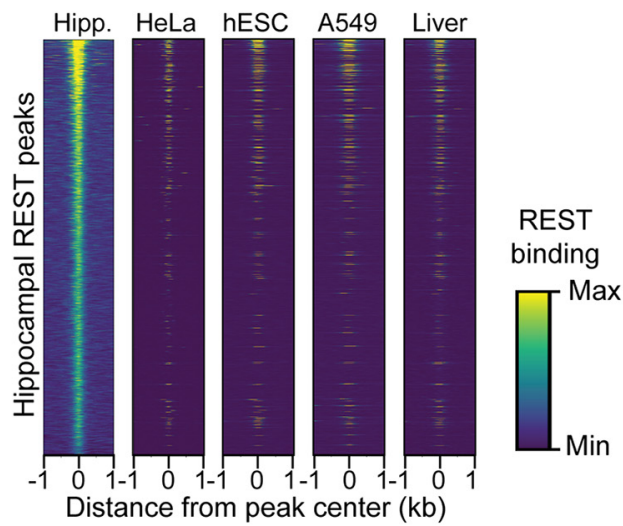

D
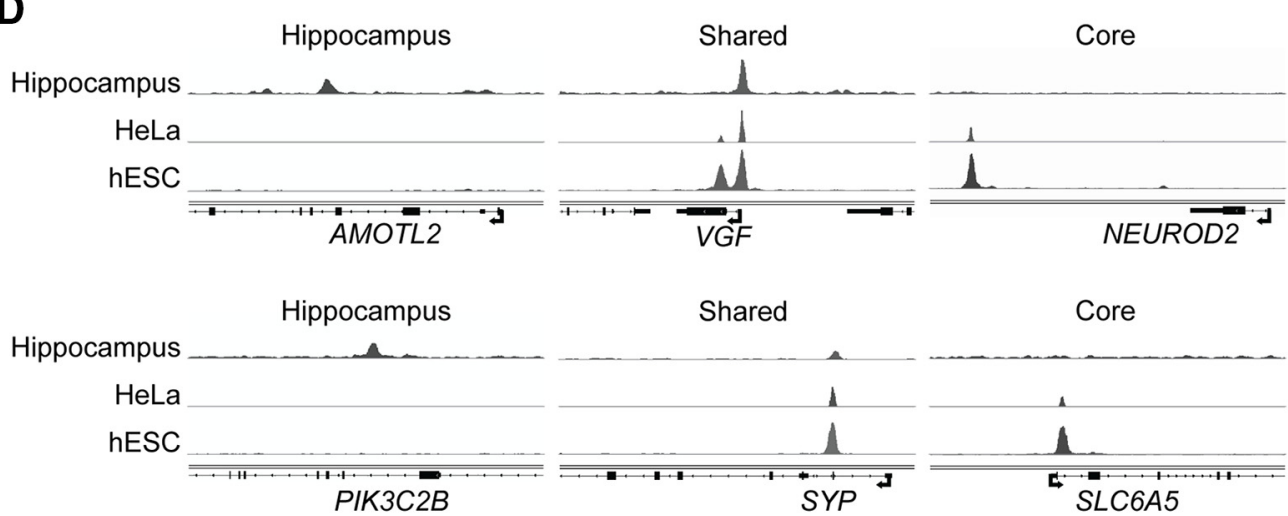

$\mathbf{E}$
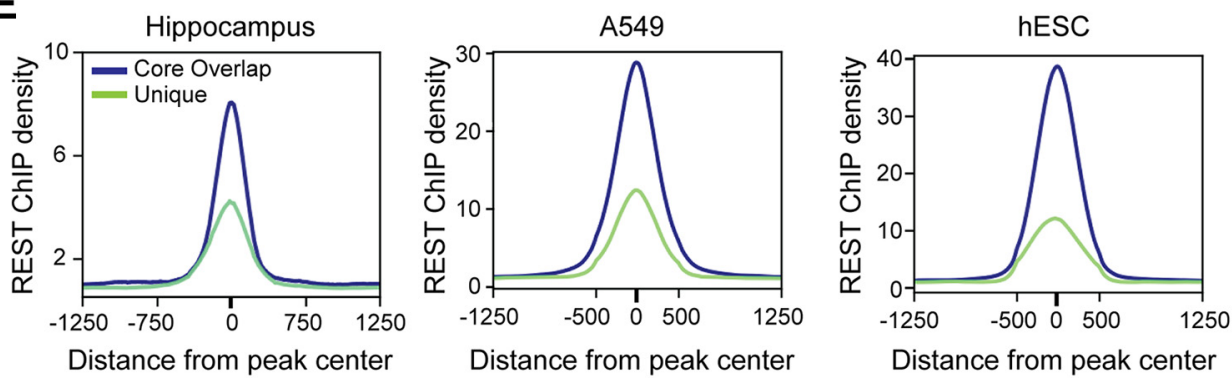

Figure 5. REST ChIP-seq peaks in human hippocampus are largely unique compared with REST peaks in non-neuronal cell types. $\boldsymbol{A}$, Venn diagram of REST peaks in human hippocampus shared with Core REST-bound peaks. A full list of human hippocampal peaks and their locations can be found in Extended Data Figure 5-1. Core peaks are defined as REST bound peaks in $>40$ of 48 ENCODE ChIP-seq replicates from human non-neuronal cell lines (Extended Data Figure 4-1). B. Heap maps representing the cell type-specific density of REST in peaks from our ChIP-seq analysis (hippocampus) relative to other Core REST ChIP-seq datasets (ENCODE). Each heatmap is scaled to the maximum level of REST bound in each tissue/cell type. C, Distribution of REST peak locations represented as a percentage of total peaks. D, REST peaks at representative genomic loci in hippocampus and indicated non-neuronal cell types, scaled to maximum REST peak height in each dataset. $\boldsymbol{E}$, Mean ChIP-seq read density plots for REST at peaks unique to a particular tissue or cell type.

\section{REST bound peaks in human hippocampus show little} overlap with REST peaks in mouse hippocampus or mouse non-neuronal cell types

Our ChIP-seq analysis indicated that the human REST binding profile, in addition to being distinct from profiles in other human cell types, was also strikingly dissimilar from mouse hippocampal binding profiles (Extended Data Fig. 5-1). First, the total number of REST peaks in human hippocampus far exceeded the number of REST peaks in murine hippocampus (2125 vs 221 peaks; Fig. $5 A$ and Fig. 2A, respectively). This result is likely related to a lower REST protein level in mouse compared
Table 2. Human REST peak number and underlying RE1 motif status for hippocampus and core cell lines ${ }^{a}$

\begin{tabular}{lccc}
\hline Tissue/cell type & With consensus RE1 & With related RE1 & Lack any RE1 \\
\hline Hippocampus unique (1886) & 41 & 1129 & 716 \\
Hippocampus and Core (239) & 189 & 49 & 1 \\
Core (2378) & 1422 & 833 & 123 \\
\hline
\end{tabular}

${ }^{{ }^{S} \text { Summary of ChIP-seq results comparing REST binding characteristics among hippocampus and Core cell lines }}$ defined as human non-neuronal ChIP-seq datasets from Encode. Numbers in parentheses indicate total peak numbers. 
with human hippocampus (Fig. 2). Second, only 9 of the 1886 peaks unique to the human hippocampus (0.5\%) were found at the corresponding location in mouse hippocampus or any murine non-neuronal cell type. Third, unlike unique peaks of mouse hippocampal ChIP, human unique peaks did not show bias toward intergenic regions (Fig. $5 C$ and Fig. $3 D$ ). Conversely, when analyzing the complete dataset of REST target genes unique to the mouse hippocampus (Extended Data Fig. 3-1), none of these genes was associated with REST peaks in human hippocampus, and only one, Gtf3c3 (General transcription factor $3 c$ polypeptide 3 ), a gene involved in neurodevelopmental disorders (Anazi et al., 2017), was also bound by REST in any of the human cell lines analyzed (Davis et al., 2018).

\section{Unique human REST-bound hippocampal sites include a new class of reactome categories related to innate immunity and inflammation signaling}

We analyzed the unique hippocampal REST-associated genes by $\mathrm{GO}$ and Reactome category enrichments (Fabregat et al., 2018) (Extended Data Fig. 6-1). Surprisingly, in contrast to the RESTassociated genes shared with the Core dataset, which were largely prototypical neuronal REST target genes, the top three Reactome enrichment categories included genes encoding proteins related to innate immunity and inflammation signaling (Fig. 6A; Extended Data Fig. 6-1; $p<0.05$, Fisher's exact test corrected for false discovery rate $[\mathrm{FDR}]$ of 0.05$)$. We tested a representative subset of these genes by quantitative ChIP (qChIP) on hippocampal tissue acquired from similarly aged individuals additional to the hippocampal tissue used for the ChIP-seq. A canonical REST target gene (GLRA1) with a consensus RE1 sequence served as a positive qChIP control. Primers for downstream sequences that lacked REST peaks served as negative controls for the REST antibody pulldown. REST binding at all but one of the ChIPseq identified genes, the Inhibitor of Nuclear factor Kappa $B$ Kinase subunit Beta $(I K B K B)$, was validated by the qChIP results (Fig. 6B).

Further computational analysis of the REST target genes highlights the novel nature of these hippocampal-specific targets. Using Enrichr, we looked for ChIP-X Enrichment Analysis (ChEA) (Lachmann et al., 2010) of our REST targets in other published ChIP-seq datasets. We found that the Core-overlapping REST targets tended to be highly enriched in other REST ChIP-seq datasets, as well as being enriched in REST cofactors, such as HDAC2, SUZ12, and EZH2 (Roopra et al., 2000; Dietrich et al., 2012) (Fig. 7A). By contrast, the hippocampal unique peaks showed higher enrichment for non-REST-regulated genes, such as those regulated by transcription factors, such as GATA1 and RUNX1 $(p<2.78 \mathrm{E}-20$ and $p<1.44 \mathrm{E}-7$; Fig. $7 A$; Extended Data Fig. 6-1).

We also sought to identify binding motifs for any other transcription factors that might recruit REST indirectly to the RESTbound sites, because $38 \%$ (716) of the unique human hippocampal peaks lacked RE1 or related sequences (Table 2). For this purpose, we applied the DIVERSITY program (Mitra et al., 2018) to all sequences underlying the hippocampal REST peaks. In addition to the conserved RE1 motifs identified previously (Fig. 3B), DIVERSITY identified three enriched motifs related to known transcription factor binding motifs (Fig. $7 B$ ). The predominant motif, detected in 663 peaks, significantly matched the motifs for the factors Sfpi1/PU.1, Prdm1, Irf1, and Etv6, as determined by the motif comparison tool, Tomtom (Gupta et al., 2007) (FDR-adjusted $p<0.05$ ). These transcription factors are all involved in the regulation of immune cell development and function (Chang et al., 2000; Tamura et al., 2008; Gupta et al., 2009; J. Wang et al., 2010), consistent with the Reactome enrichments. Further, the two other identified motifs matched the binding sites for the transcription factors Sp2, Runx1, and Gfilb, which regulate immune system differentiation (Growney et al., 2005; van der Meer et al., 2010; Terrados et al., 2012) (Fig. 7B). It seems possible that these factors are in complexes with REST, indirectly recruiting REST to the peak 
A

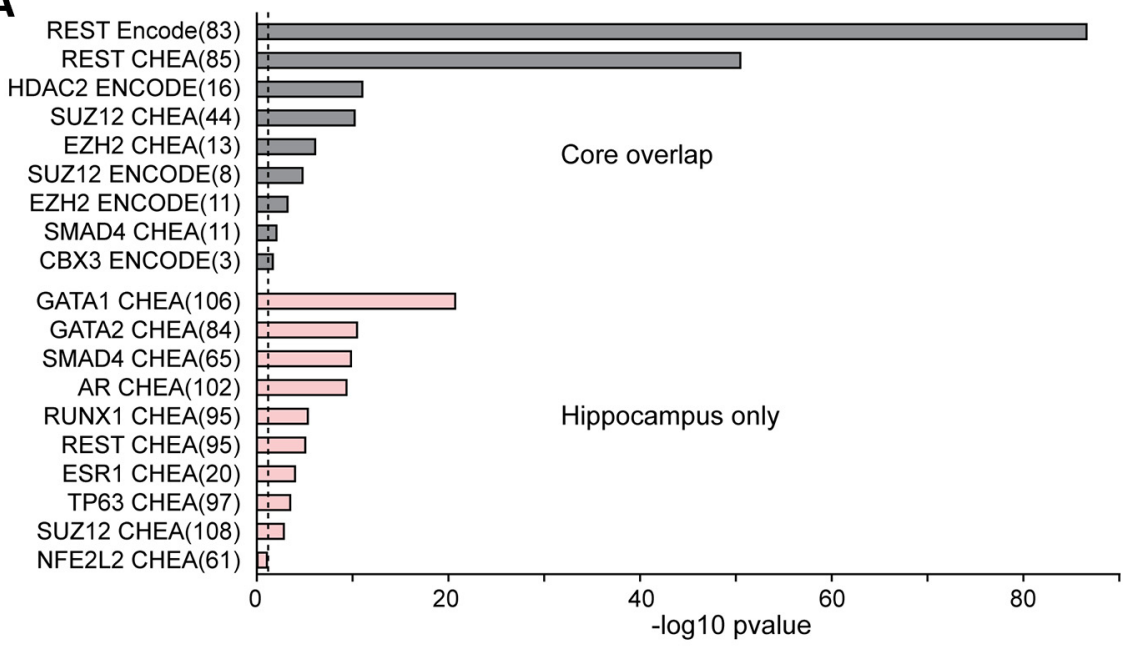

B
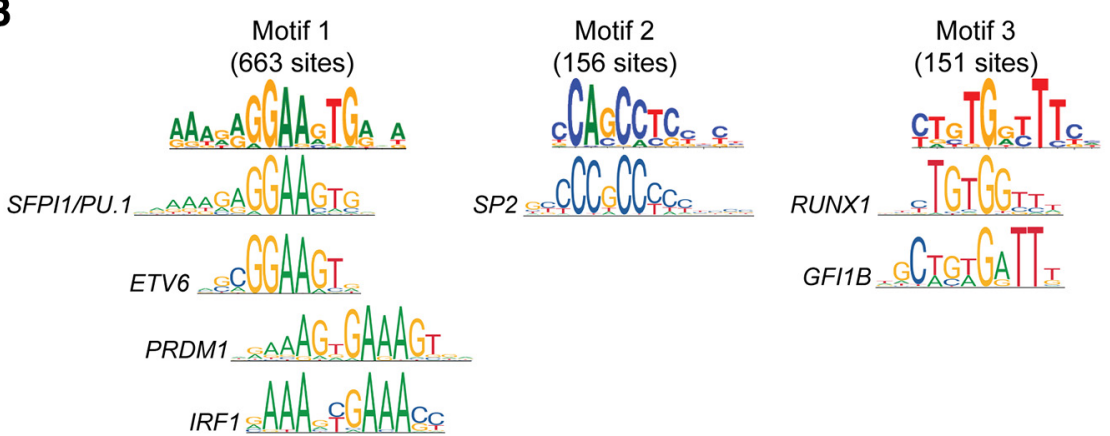

Figure 7. Human hippocampal-specific REST peaks are distinct from canonical REST targets and show an enrichment with immune-related transcriptional factors and non-RE1 binding motifs. $A$, Enrichr transcription factor enrichment for genes containing REST peaks unique to either Core or hippocampus using the ENCODE/ChEA Consensus TF database (Lachmann et al., 2010). Genes common to both hippocampal and Core datasets were highly enriched for REST and previously reported REST cofactors (HDAC, SUZ12), whereas genes unique to the hippocampus had a distinct profile with relatively low REST enrichment. Dashed line indicates $p=0.05$. $\boldsymbol{B}$, Non-RE1 motifs present in the REST peak dataset unique to the human hippocampus. Motifs were identified by DIVERSITY de novo motif analysis. Top, Consensus motifs found in REST peaks. Bottom, Transcription factors that bind to related sequences. The number of sites refers to the number of REST peaks that are associated with a motif. Motifs were based on Tomtom motif analysis (Gupta et al., 2007) FDR-adjusted $p<0.05$.

loci to mediate repression. This interpretation is supported by previous studies that show the REST corepressor, CoREST, in complexes with PU.1, Runx1, and Gfilb (Andrés et al., 1999; Gu et al., 2014; Yamamoto et al., 2018). However, additional experiments testing whether REST is in complexes with the immune transcription factors in hippocampus are required for confirmation.

\section{The innate immunity REST target genes are likely repressed in astrocytes}

A recent single-cell transcriptomic analysis indicated that REST transcript numbers were low overall and distributed differentially within the adult hippocampus, but highest in the astrocyte population (males aged 40-65 years) (Habib et al., 2017). Thus, these data point strongly toward glia as a potential major source of our genome-wide REST binding sites. Although REST transcripts were present at higher levels in neural stem cells than in the other glial subtypes, the stem cells were represented in a smaller proportion of the total cells (Y. Zhang et al., 2016) (Fig. 8A,B). The finding that glia constitute the largest proportion of cell types in the human hippocampus provides more support for the idea that non-neuronal cells are the most likely source of our ChIP-seq data, and immunohistochemistry supports REST protein in both glia and neurons in adult hippocampus (Fig. 8B,C).

To determine whether the RESTbound innate immunity target genes were repressed in the glia, we relied on the only available RNA-seq analysis derived by immuno-panning cell types from acutely dissociated human cortices (Y. Zhang et al., 2016). A previous study indicated similar REST protection of neurodegeneration in human cortex and hippocampus (Lu et al., 2014) and REST levels increase in both brain regions with age. Using this dataset, we parsed genes within each Reactome category (Fig. 6A) according to whether they were or were not hippocampal REST target genes. We then compared the expression levels of the two groups in astrocytes, oligodendrocytes, and microglia. Consistent with REST repression in astrocytes, genes associated with REST binding were expressed at significantly lower levels $(p<0.05$, Mann-Whitney test) in this cell type in the categories innate immune system, immune system, neutrophil degranulation, and signaling by interleukins, compared with the genes lacking REST binding peaks (Fig. 8D). The results were largely recapitulated using other single-cell datasets (Darmanis et al., 2015; D. Wang et al., 2018). There was no evidence for statistically significant repression of these genes in the other cell types. Because REST is a repressor, expression levels of the target genes in other cell types may be more dependent on activity of cell-specific enhancers. For example, microglia, which have a hematopoietic origin, represent $15 \%$ of the cells in hippocampus and express the highest level of REST RNA. Yet, target gene repression was not observed in microglia, perhaps because of myeloid-specific enhancers. More refined analyses in hippocampus are required to draw conclusions in the other neuronal and non-neuronal cell types. Interestingly, mouse REST is distributed similarly to human REST in terms of non-neuronal versus neuronal cell types (Fig. $8 A$ ), except for expression in neural progenitors, where its level is higher for human REST and represents a larger proportion of the cell types.

\section{Discussion}

Here, we have compared, for the first time, the genome-wide REST binding profiles in mouse and human hippocampus. We found that mouse and human were highly dissimilar in several ways. They differed in terms of REST expression profiles with age, in the total number of REST binding sites, and in the nature of the associated bound genes, raising questions about how well mouse postnatal development and aging model the same processes in human brain. We also identified new gene classes associated with REST binding that are unique to the human hippocampus from all other cell types, as well as mouse 
A

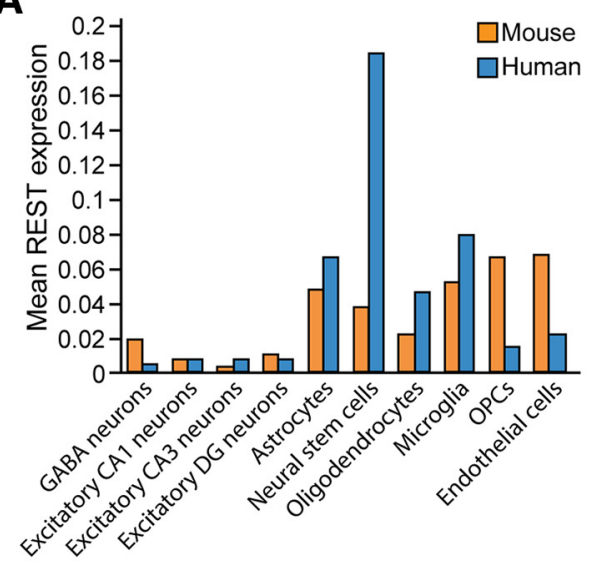

B

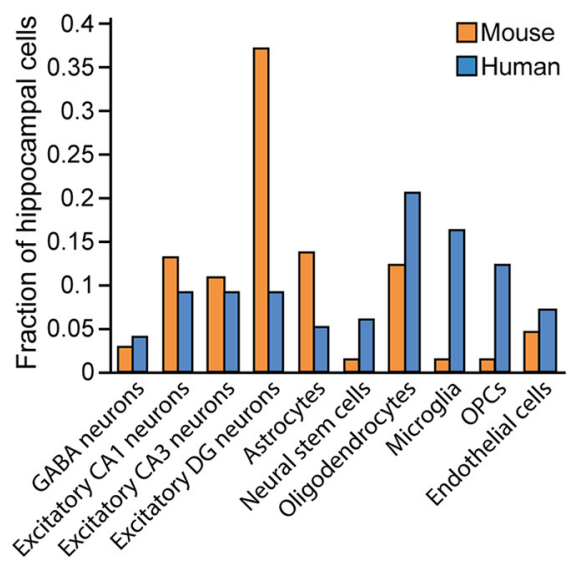

C

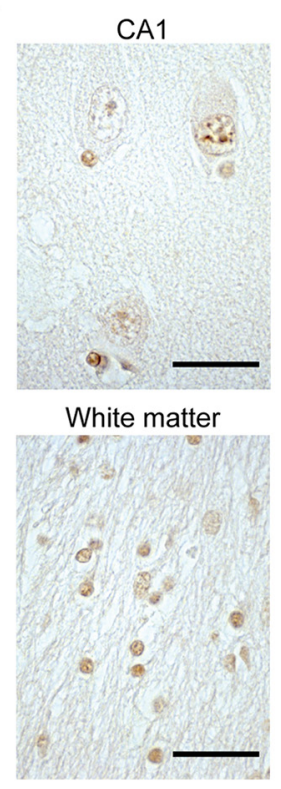

D

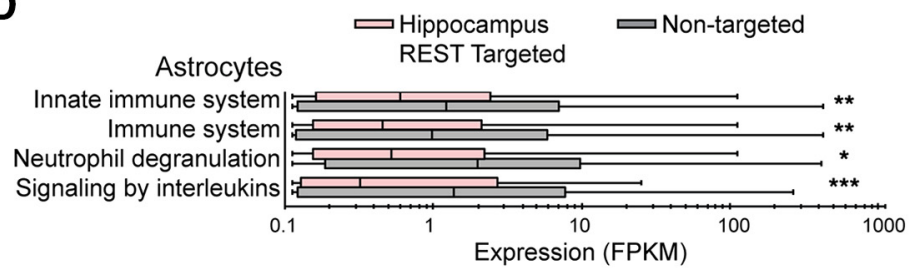

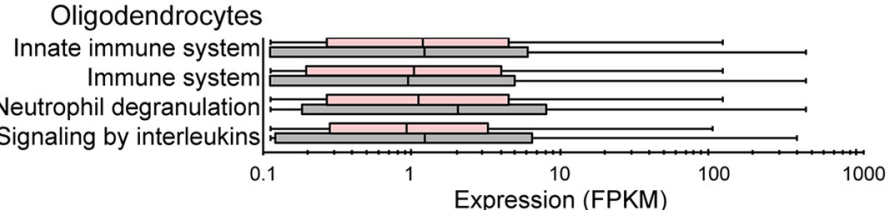

Microglia

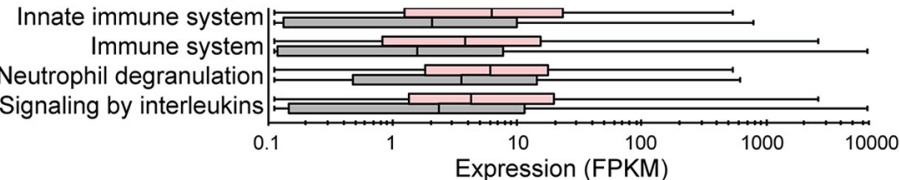

Figure 8. The predominant cell source of REST ChIP-seq data in human hippocampus is likely glia. $\boldsymbol{A}$, REST transcript expression levels from single-cell RNA-seq from mouse and human hippocampi. Data adapted from Habib et al. (2017). B, Proportion of cells expressing REST transcripts in mouse and human hippocampi. Data adapted from Habib et al. (2017). C, Immunolabeling of human hippocampal pyramidal and granular sectors (top) depicts pyramidal neurons (large cells) and associated satellite glia (small cells) with nuclear REST expression in the CA1 sector of the hippocampus. Oligodendroglial temporal white matter (bottom) shows REST expression in the white matter of the temporal lobe, which is devoid of neurons; the large majority of cells are oligodendroglia (smaller round nuclei); rare larger nuclei belong to astrocytes. Higher-magnification staining shows REST in both neurons and glia that is almost exclusively nuclear. Scale bar, $30 \mu \mathrm{m}$. D, Box-and-whisker plots showing expression levels from human cortical cell types (Zhang et al., 2016) for Reactome ontology categories (Fabregat et al., 2018), parsed by whether they are targets of REST in hippocampus (pink) or untargeted (gray). Whiskers represent minimum and maximum. Boxes represent first quartile to median, and median to third quartile. ${ }^{*} p<0.05 ;{ }^{* *} p<0.01 ;{ }^{* * *} p<0.001$; Mann-Whitney test. Gene expression values adapted from Zhang et al. (2016).

hippocampus, representing largely, but not exclusively, processes related to innate immunity and inflammatory signaling. Finally, we leveraged public single-cell transcriptomic databases and immunohistochemistry to highlight a role for glial REST in potentially maintaining human brain health through the repression of immune and inflammatory responses.

We performed the ChIP-seq analysis in mouse when, at 5 weeks of age, REST levels were still robust enough for analysis and at a similar level of REST in human hippocampus. A proportion of the target genes represented canonical neuronal genes that are repressed in stem/neuronal progenitors as well as in non-neuronal cells within the CNS (e.g., glia) and muscle cells. This observation is consistent with the historical function of REST in repressing murine neuronal genes. Further, two recent single-cell transcriptomic analyses in similarly aged mice indicated that the highest expression of REST was in the glia and neural progenitors, with much lower expression in the neurons.
The target genes we identified as unique to the hippocampus were largely because of binding of REST to the Tle genes. Not all paralogs of the Tle gene family have been studied in mouse brain, but at least two of them function in regulating early neurodevelopment (Roth et al., 2010; Mukherjee et al., 2016). A previous study identified the murine tle3 gene as upregulated in a REST shRNA knockdown experiment in adult quiescent neural progenitors in culture (Mukherjee et al., 2016). This finding lends further support for our suggestion that neural progenitors are one of the sources of our ChIP-seq data and confirms the predicted repressive function of REST. The lack of other REST target genes that overlapped with this study may point to differences in culture or a different role for REST in stem/progenitors in this hippocampal niche later in life.

In contrast to the limited unique REST target genes we identified in the mouse hippocampus, we identified numerous REST 
peaks and target genes from human hippocampus, including a substantial number of target genes unique to this brain region. Similar to findings from previous studies, a large percentage (38\%) of human REST peaks were associated with sequences lacking any variation of the RE1 sequence (R. Johnson et al., 2009; Rockowitz and Zheng, 2015) (Table 2). Of the human hippocampal REST peaks that had no orthologous mouse sequence (462 peaks), a majority (54\%) were associated with both consensus and nonconsensus RE1 sequences. This result suggests that newly evolved RE1 sequences account for a large proportion of REST binding site expansion in human, and adds to previous results in hESCs where consensus RE1 sequences with nonconserved REST peaks were primate or human-specific (R. Johnson et al., 2009; Rockowitz and Zheng, 2015).

A recent single-cell RNA-seq analysis of mouse and human hippocampus indicated low overall expression of REST transcripts in all cell types. The low RNA expression is consistent with the weak immunostaining of both glia and neurons in postmortem hippocampal sections (Fig. 8C). In both species, REST was expressed to the highest level in non-neuronal cell types, predominantly glia in human hippocampus and neural stem cells in mouse. However, while glia represented the highest proportion of cell types in human hippocampus (Habib et al., 2017; Saunders et al., 2018) (Fig. 1C,D), neurons occupied this position in mouse, suggesting that REST may play a disproportionately important role in human hippocampus. The potential difference in REST function in mouse and human based on expression profiles and cell proportionality is consistent with our ChIPseq results indicating minimal overlap between REST target genes in human and mouse hippocampus. However, as we did not examine other mammalian rodent brains, for example, rat hippocampus, we cannot exclude the possibility that mammals other than mouse may share brain REST-bound genes with human REST.

REST has been studied experimentally from the perspective of human aging and age-related disease, particularly in the context of AD (Lu et al., 2014). For example, it was reported that REST levels continue to increase with human age, in neurons in both cortex and hippocampus, while levels are decreased in AD tissue. Interestingly, in $\mathrm{Lu}$ et al. (2014), the authors identified a set of REST repressed genes in neurons encoding proteins that promote apoptosis and $\mathrm{AD}$ pathology, concentrating primarily on human prefrontal cortex, but also showing a correlation of REST levels and AD pathology in hippocampus as well. In our ChIP-seq analysis of postmortem hippocampus, we confirmed increased REST expression with age, but we identified a different set of REST target genes. This difference may be because of the heterogeneity of cell types in intact hippocampus, and our data suggesting that glia are the most likely major source of our ChIPseq REST peaks. Although our REST target genes were different, they did encode proteins related to preserving brain health. In our group, several of the qChIP-validated genes relating to innate immunity have been implicated in $\mathrm{AD}$ (Fig. 6B; Extended Data Fig. 6-1) and would be predicted to increase with reduced levels of REST (Lu et al., 2014). For example, Arachidonate 5Lipoxygenase (ALOX5), an enzyme that converts arachidonic acid to leukotrienes, is expressed to abnormally high levels in $\mathrm{AD}$ in human brains (Firuzi et al., 2008). Interestingly, we also identified Coactosin-like protein (COTL1) as a REST target gene. Some evidence supports this protein as an ALOX5 chaperone and increased COTL1 expression in microglia is associated with AD neuropathology (Rangaraju et al., 2018). Expression of the human REST target gene, Diaphanous Related Formin 1 (DIAPH1), involved in actin polymerization, is increased in neurons and glia in AD (Derk et al., 2018).

Together, including studies indicating an association of human REST single nucleotide polymorphisms with AD (Mozzi et al., 2017), accumulating data points to a role for REST levels in protecting epigenetic homeostasis in long-lived neural cells. This protection may extend to other forms of neurodegeneration as well, including Parkinson's disease and multiple sclerosis, which have been associated with inflammasome complexes. NLR Family Pyrin Domain Containing protein 4, a component of inflammatory complexes, is encoded by one of the REST target genes in our analysis and merits further study.

To more definitively clarify the cellular basis of REST repression in human brain, REST ChIP-seq analyses, or more sensitive methods, are required on sorted cells from brain regions, for coordination with RNA-seq and single-cell ATAC analyses (Nativio et al., 2018; Xu et al., 2018; Meers et al., 2019; Ramani et al., 2019). These studies will also open the door to mechanistic studies to address fundamental gaps in our general knowledge of repressors, such as precisely how REST is recruited to genes lacking an RE1 sequence, and, conversely, how REST is prevented from binding in neurons to the consensus RE1 sequences in the prototypical gene targets related to neuronal function. Finally, the function of REST in human glia is still ill-defined but likely to contribute importantly to protecting human brain longevity.

\section{References}

Aigner S, Yeo GW (2009) Terminal differentiation: REST. In: SquireLR (ed), pp 921-927. Encyclopedia of neuroscience. San Diego: Academic.

Anazi S, Maddirevula S, Salpietro V, Asi YT, Alsahli S, Alhashem A, Shamseldin HE, AlZahrani F, Patel N, Ibrahim N, Abdulwahab FM, Hashem M, Alhashmi N, Al Murshedi F, Al Kindy A, Alshaer A, Rumayyan A, Al Tala S, Kurdi W, Alsaman A, et al. (2017) Expanding the genetic heterogeneity of intellectual disability. Hum Genet 136:14191429.

Andrés ME, Burger C, Peral-Rubio MJ, Battaglioli E, Anderson ME, Grimes J, Dallman J, Ballas N, Mandel G (1999) CoREST: a functional corepressor required for regulation of neural-specific gene expression. Proc Natl Acad Sci USA 96:9873-9878.

Bailey TL (2011) DREME: motif discovery in transcription factor ChIP-seq data. Bioinformatics 27:1653-1659.

Ballas N, Grunseich C, Lu DD, Speh JC, Mandel G (2005) REST and its corepressors mediate plasticity of neuronal gene chromatin throughout neurogenesis. Cell 121:645-657.

Ballas N, Mandel G (2005) The many faces of REST oversee epigenetic programming of neuronal genes. Curr Opin Neurobiol 15:500-506.

Bruce AW, Donaldson IJ, Wood IC, Yerbury SA, Sadowski MI, Chapman M, Göttgens B, Buckley NJ (2004) Genome-wide analysis of repressor element 1 silencing transcription factor/neuron-restrictive silencing factor (REST/NRSF) target genes. Proc Natl Acad Sci USA 101:10458-10463.

Calderone A, Jover T, Noh K, Tanaka H, Yokota H, Lin Y, Grooms SY, Regis R, Bennett MV, Zukin RS (2003) Ischemic insults derepress the gene silencer REST in neurons destined to die. J Neurosci 23:2112-2121.

Chang DH, Angelin-Duclos C, Calame K (2000) BLIMP-1: trigger for differentiation of myeloid lineage. Nat Immunol 1:169-176.

Chen GL, Miller GM (2018) Alternative REST splicing underappreciated. eNeuro 5:ENEURO.0034-18.2018.

Chen RA, Stempor P, Down TA, Zeiser E, Feuer SK, Ahringer J (2014) Extreme HOT regions are $\mathrm{CpG}$-dense promoters in C. elegans and humans. Genome Res 24:1138-1146.

Chen EY, Tan CM, Kou Y, Duan Q, Wang Z, Meirelles GV, Clark NR, Ma'ayan A (2013) Enrichr: interactive and collaborative HTML5 gene list enrichment analysis tool. BMC Bioinformatics 14:128.

Chong JA, Tapia-Ramírez J, Kim S, Toledo-Aral JJ, Zheng Y, Boutros MC, Altshuller YM, Frohman MA, Kraner SD, Mandel G (1995) REST: a mammalian silencer protein that restricts sodium channel gene expression to neurons. Cell 80:949-957. 
Darmanis S, Sloan SA, Zhang Y, Enge M, Caneda C, Shuer LM, Hayden Gephart MG, Barres BA, Quake SR (2015) A survey of human brain transcriptome diversity at the single cell level. Proc Natl Acad Sci USA 112:7285-7290.

Davis CA, Hitz BC, Sloan CA, Chan ET, Davidson JM, Gabdank I, Hilton JA, Jain K, Baymuradov UK, Narayanan AK, Onate KC, Graham K, Miyasato SR, Dreszer TR, Strattan JS, Jolanki O, Tanaka FY, Cherry JM (2018) The Encyclopedia of DNA Elements (ENCODE): data portal update. Nucleic Acids Res 46:D794-D801.

Derk J, Bermudez Hernandez K, Rodriguez M, He M, Koh H, Abedini A, Li H, Fenyö D, Schmidt AM (2018) Diaphanous 1 (DIAPH1) is highly expressed in the aged human medial temporal cortex and upregulated in myeloid cells during Alzheimer's disease. J Alzheimers Dis 64:995-1007.

Dietrich N, Lerdrup M, Landt E, Agrawal-Singh S, Bak M, Tommerup N, Rappsilber J, Södersten E, Hansen K (2012) REST-mediated recruitment of polycomb repressor complexes in mammalian cells. PLoS Genet 8: e1002494.

Fabregat A, Jupe S, Matthews L, Sidiropoulos K, Gillespie M, Garapati P, Haw R, Jassal B, Korninger F, May B, Milacic M, Roca CD, Rothfels K, Sevilla C, Shamovsky V, Shorser S, Varusai T, Viteri G, Weiser J, Wu G, et al. (2018) The Reactome Pathway Knowledgebase. Nucleic Acids Res 46:D649-D655.

Firuzi O, Zhuo J, Chinnici CM, Wisniewski T, Praticò D (2008) 5Lipoxygenase gene disruption reduces amyloid-beta pathology in a mouse model of Alzheimer's disease. FASEB J 22:1169-1178.

Gao Z, Ure K, Ding P, Nashaat M, Yuan L, Ma J, Hammer RE, Hsieh J (2011) The master negative regulator REST/NRSF controls adult neurogenesis by restraining the neurogenic program in quiescent stem cells. J Neurosci 31:9772-9786.

Grimes JA, Nielsen SJ, Battaglioli E, Miska EA, Speh JC, Berry DL, Atouf F, Holdener BC, Mandel G, Kouzarides T (2000) The co-repressor mSin3A is a functional component of the REST-CoREST repressor complex. J Biol Chem 275:9461-9467.

Growney JD, Shigematsu H, Li Z, Lee BH, Adelsperger J, Rowan R, Curley DP, Kutok JL, Akashi K, Williams IR, Speck NA, Gilliland DG (2005) Loss of Runx1 perturbs adult hematopoiesis and is associated with a myeloproliferative phenotype. Blood 106:494-504.

Gu X, Hu Z, Ebrahem Q, Crabb JS, Mahfouz RZ, Radivoyevitch T, Crabb JW, Saunthararajah Y (2014) Runxl regulation of Pu.1 corepressor/coactivator exchange identifies specific molecular targets for leukemia differentiation therapy. J Biol Chem 289:14881-14895.

Gupta P, Gurudutta GU, Saluja D, Tripathi RP (2009) PU.1 and partners: regulation of haematopoietic stem cell fate in normal and malignant haematopoiesis. J Cell Mol Med 13:4349-4363.

Gupta S, Stamatoyannopoulos JA, Bailey TL, Noble WS (2007) Quantifying similarity between motifs. Genome Biol 8:R24.

Habib N, Avraham-Davidi I, Basu A, Burks T, Shekhar K, Hofree M, Choudhury SR, Aguet F, Gelfand E, Ardlie K, Weitz DA, RozenblattRosen O, Zhang F, Regev A (2017) Massively parallel single-nucleus RNA-seq with DroNc-seq. Nat Methods 14:955-958.

Hansen J, Floss T, Van Sloun P, Füchtbauer EM, Vauti F, Arnold HH, Schnütgen F, Wurst W, von Melchner H, Ruiz P (2003) A large-scale, gene-driven mutagenesis approach for the functional analysis of the mouse genome. Proc Natl Acad Sci USA 100:9918-9922.

He P, Williams BA, Trout D (2020) The changing mouse embryo transcriptome at whole tissue and single-cell resolution. bioRxiv 150599.

Heinz S, Benner C, Spann N, Bertolino E, Lin YC, Laslo P, Cheng JX, Murre C, Singh H, Glass CK (2010) Simple combinations of lineage-determining transcription factors prime cis-regulatory elements required for macrophage and B cell identities. Mol Cell 38:576-589.

Hu XL, Cheng X, Cai L, Tan GH, Xu L, Feng XY, Lu TJ, Xiong H, Fei J, Xiong ZQ (2011) Conditional deletion of NRSF in forebrain neurons accelerates epileptogenesis in the kindling model. Cereb Cortex 21:21582165.

Jain D, Baldi S, Zabel A, Straub T, Becker PB (2015) Active promoters give rise to false positive 'Phantom Peaks' in ChIP-seq experiments. Nucleic Acids Res 43:6959-6968.

Johnson DS, Mortazavi A, Myers RM, Wold B (2007) Genome-wide mapping of in-vivo protein-DNA interactions. Science 316:1497-1502.

Johnson R, Samuel J, Ng CK, Jauch R, Stanton LW, Wood IC (2009) Evolution of the vertebrate gene regulatory network controlled by the transcriptional repressor REST. Mol Biol Evol 26:1491-1507.
Kaneko N, Hwang JY, Gertner M, Pontarelli F, Zukin RS (2014) Casein kinase 1 suppresses activation of REST in insulted hippocampal neurons and halts ischemia-induced neuronal death. J Neurosci 34:6030-6039.

Kraner SD, Chong JA, Tsay HJ, Mandel G (1992) Silencing the type II sodium channel gene: a model for neural-specific gene regulation. Neuron 9:37-44.

Krebs W, Schmidt SV, Goren A, De Nardo D, Labzin L, Bovier A, Ulas T, Theis H, Kraut M, Latz E, Beyer M, Schultze JL (2014) Optimization of transcription factor binding map accuracy utilizing knockout-mouse models. Nucleic Acids Res 42:13051-13060.

Kuwabara T, Hsieh J, Nakashima K, Taira K, Gage FH (2004) A small modulatory dsRNA specifies the fate of adult neural stem cells. Cell 116:779793.

Lachmann A, Xu H, Krishnan J, Berger SI, Mazloom AR, Ma’ayan A (2010) ChEA: transcription factor regulation inferred from integrating genomewide ChIP-X experiments. Bioinformatics 26:2438-2444.

Lu T, Aron L, Zullo J, Pan Y, Kim H, Chen Y, Yang TH, Kim HM, Drake D, Liu XS, Bennett DA, Colaiácovo MP, Yankner BA (2014) REST and stress resistance in ageing and Alzheimer's disease. Nature 507:448-454.

McClelland S, Brennan GP, Dubé C, Rajpara S, Iyer S, Richichi C, Bernard C, Baram TZ (2014) The transcription factor NRSF contributes to epileptogenesis by selective repression of a subset of target genes. Elife 3:e01267.

McGann JC, Oyer JA, Garg S, Yao H, Liu J, Feng X, Liao L, Yates JR, Mandel G (2014) Polycomb- and REST-associated histone deacetylases are independent pathways toward a mature neuronal phenotype. Elife 3:e04235.

Meers MP, Bryson TD, Henikoff JG, Henikoff S (2019) Improved CUT\&RUN chromatin profiling tools. Elife 8:e46314.

Mi H, Muruganujan A, Ebert D, Huang X, Thomas PD (2019) PANTHER version 14: more genomes, a new PANTHER GO-slim and improvements in enrichment analysis tools. Nucleic Acids Res 47:D419-D426.

Mitra S, Biswas A, Narlikar L (2018) DIVERSITY in binding, regulation, and evolution revealed from high-throughput ChIP. PLoS Comput Biol 14: e1006090.

Mortazavi A, Leeper Thompson EC, Garcia ST, Myers RM, Wold B (2006) Comparative genomics modeling of the NRSF/REST repressor network: from single conserved sites to genome-wide repertoire. Genome Res $16: 1208-1221$.

Mozzi A, Guerini FR, Forni D, Costa AS, Nemni R, Baglio F, Cabinio M, Riva S, Pontremoli C, Clerici M, Sironi M, Cagliani R (2017) REST, a master regulator of neurogenesis, evolved under strong positive selection in humans and in non human primates. Sci Rep 7:9530.

Mukherjee S, Brulet R, Zhang L, Hsieh J (2016) REST regulation of gene networks in adult neural stem cells. Nat Commun 7:13360.

Nativio R, Donahue G, Berson A, Lan Y, Amlie-Wolf A, Tuzer F, Toledo JB, Gosai SJ, Gregory BD, Torres C, Trojanowski JQ, Wang LS, Johnson FB, Bonini NM, Berger SL (2018) Dysregulation of the epigenetic landscape of normal aging in Alzheimer's disease [published correction appears in Nat Neurosci. 2018; 21:1018]. Nat Neurosci 21:497-505.

Nechiporuk T, McGann J, Mullendorff K, Hsieh J, Wurst W, Floss T, Mandel G (2016) The REST remodeling complex protects genomic integrity during embryonic neurogenesis. Elife 5:e09584.

Nguyen MT, Mattek N, Woltjer R, Howieson D, Silbert L, Hofer S, Kaye J, Dodge H, Erten-Lyons D (2018) Pathologies underlying longitudinal cognitive decline in the oldest old. Alzheimer Dis Assoc Disord 32:265-269.

Noh KM, Hwang JY, Follenzi A, Athanasiadou R, Miyawaki T, Greally JM, Bennett MV, Zukin RS (2012) Repressor element-1 silencing transcription factor (REST)-dependent epigenetic remodeling is critical to ischemia-induced neuronal death. Proc Natl Acad Sci USA 109:E962-E971.

Otto SJ, McCorkle SR, Hover J, Conaco C, Han JJ, Impey S, Yochum GS, Dunn JJ, Goodman RH, Mandel G (2007) A new binding motif for the transcriptional repressor REST uncovers large gene networks devoted to neuronal functions. J Neurosci 27:6729-6739.

Palm K, Belluardo N, Metsis M, Timmusk T (1998) Neuronal expression of zinc finger transcription factor REST/NRSF/XBR gene. J Neurosci 18:1280-1296.

Park D, Lee Y, Bhupindersingh G, Iyer VR (2013) Widespread misinterpretable ChIP-seq bias in yeast. PLoS One 8:e83506.

Ramani V, Qiu R, Shendure J (2019) High sensitivity profiling of chromatin structure by MNase-SSP. Cell Rep 26:2465-2476.e4.

Rangaraju S, Dammer EB, Raza SA, Gao T, Xiao H, Betarbet R, Duong DM, Webster JA, Hales CM, Lah JJ, Levey AI, Seyfried NT (2018) Quantitative 
proteomics of acutely-isolated mouse microglia identifies novel immune Alzheimer's disease-related proteins. Mol Neurodegener 13:34.

Rockowitz S, Lien WH, Pedrosa E, Wei G, Lin M, Zhao K, Lachman HM, Fuchs E, Zheng D (2014) Comparison of REST cistromes across human cell types reveals common and context-specific functions. PLoS Comput Biol 10:e1003671.

Rockowitz S, Zheng D (2015) Significant expansion of the REST/NRSF cistrome in human versus mouse embryonic stem cells: potential implications for neural development. Nucleic Acids Res 43:5730-5743.

Rodríguez CI, Buchholz F, Galloway J, Sequerra R, Kasper J, Ayala R, Stewart AF, Dymecki SM (2000) High-efficiency deleter mice show that FLPe is an alternative to Cre-loxP. Nat Genet 25:139-140.

Roopra A, Sharling L, Wood IC, Briggs T, Bachfischer U, Paquette AJ, Buckley NJ (2000) Transcriptional repression by neuron-restrictive silencer factor is mediated via the Sin3-histone deacetylase complex. Mol Cell Biol 20:2147-2157.

Roth M, Bonev B, Lindsay J, Lea R, Panagiotaki N, Houart C, Papalopulu N (2010) FoxG1 and TLE2 act cooperatively to regulate ventral telencephalon formation. Development 137:1553-1562.

Saunders A, Macosko EZ, Wysoker A, Goldman M, Krienen FM, de Rivera H, Bien E, Baum M, Bortolin L, Wang S, Goeva A, Nemesh J, Kamitaki N, Brumbaugh S, Kulp D, McCarroll SA (2018) Molecular diversity and specializations among the cells of the adult mouse brain. Cell 174:10151030.e16.

Schindelin J, Arganda-Carreras I, Frise E, Kaynig V, Longair M, Pietzsch T, Preibisch S, Rueden C, Saalfeld S, Schmid B, Tinevez JY, White DJ, Hartenstein V, Eliceiri K, Tomancak P, Cardona A (2012) Fiji: an opensource platform for biological-image analysis. Nat Methods 9:676-682.

Schoenherr CJ, Anderson DJ (1995) The neuron-restrictive silencer factor (NRSF): a coordinate repressor of multiple neuron-specific genes. Science 267:1360-1363.

Schoenherr CJ, Paquette AJ, Anderson DJ (1996) Identification of potential target genes for the neuron-restrictive silencer factor. Proc Natl Acad Sci USA 93:9881-9886

Semple BD, Blomgren K, Gimlin K, Ferriero DM, Noble-Haeusslein LJ (2013) Brain development in rodents and humans: identifying benchmarks of maturation and vulnerability to injury across species. Prog Neurobiol 106-107:1-16.

Singh-Taylor A, Molet J, Jiang S, Korosi A, Bolton JL, Noam Y, Simeone K, Cope J, Chen Y, Mortazavi A, Baram TZ (2018) NRSF-dependent epigenetic mechanisms contribute to programming of stress-sensitive neurons by neonatal experience, promoting resilience. Mol Psychiatry 23:648657.

Sun YM, Greenway DJ, Johnson R, Street M, Belyaev ND, Deuchars J, Bee T, Wilde S, Buckley NJ (2005) Distinct profiles of REST interactions with its target genes at different stages of neuronal development [published correction appears in Mol Cell Biol 16:5630-5638]
Tamura T, Yanai H, Savitsky D, Taniguchi T (2008) The IRF family transcription factors in immunity and oncogenesis. Annu Rev Immunol 26:535-584.

Terrados G, Finkernagel F, Stielow B, Sadic D, Neubert J, Herdt O, Krause M, Scharfe M, Jarek M, Suske G (2012) Genome-wide localization and expression profiling establish Sp2 as a sequence-specific transcription factor regulating vitally important genes. Nucleic Acids Res 40:7844-7857.

Teytelman L, Thurtle DM, Rine J, van Oudenaarden A (2013) Highly expressed loci are vulnerable to misleading ChIP localization of multiple unrelated proteins. Proc Natl Acad Sci USA 110:18602-18607.

van der Meer LT, Jansen JH, van der Reijden BA (2010) Gfil and Gfilb: key regulators of hematopoiesis. Leukemia 24:1834-1843.

Wang D, Liu S, Warrell J, Won H, Shi X, Navarro FC, Clarke D, Gu M, Emani P, Yang YT, Xu M, Gandal MJ, Lou S, Zhang J, Park JJ, Yan C, Rhie SK, Manakongtreecheep K, Zhou H, Nathan A, et al. (2018) Comprehensive functional genomic resource and integrative model for the human brain. Science 362:eaat8464.

Wang J, Dayyani F, Brynzka C, Sweetser D (2010) TLEs regulate myeloid proliferation and differentiation in association with modulation of NF$\kappa \mathrm{B}$ and Wnt signaling. Blood 116:4176-4176.

Wreczycka K, Franke V, Uyar B, Wurmus R, Bulut S, Tursun B, Akalin A (2019) HOT or not: examining the basis of high-occupancy target regions. Nucleic Acids Res 47:5735-5745.

Xu X, Stoyanova EI, Lemiesz AE, Xing J, Mash DC, Heintz N (2018) Species and cell-type properties of classically defined human and rodent neurons and glia. Elife 7:e37551.

Yamamoto R, Kawahara M, Ito S, Satoh J, Tatsumi G, Hishizawa M, Suzuki T, Andoh A (2018) Selective dissociation between LSD1 and GFI1B by a LSD1 inhibitor NCD38 induces the activation of ERG super-enhancer in erythroleukemia cells. Oncotarget 9:21007-21021.

Yu G, Wang LG, Han Y, He QY (2012) clusterProfiler: an R package for comparing biological themes among gene clusters. OMICS 16:284-287.

Yu G, Wang LG, He QY (2015) ChIPseeker: an R/Bioconductor package for ChIP peak annotation, comparison and visualization. Bioinformatics 31:2382-2383.

Zhang D, Wang Y, Lu P, Wang P, Yuan X, Yan J, Cai C, Chang CP, Zheng D, Wu B, Zhou B (2017) REST regulates the cell cycle for cardiac development and regeneration. Nat Commun 8:1979.

Zhang Y, Sloan SA, Clarke LE, Caneda C, Plaza CA, Blumenthal PD, Vogel H, Steinberg GK, Edwards MS, Li G, Duncan JA, Cheshier SH, Shuer LM, Chang EF, Grant GA, Gephart MG, Barres BA (2016) Purification and characterization of progenitor and mature human astrocytes reveals transcriptional and functional differences with mouse. Neuron 89:37-53.

Zullo JM, Drake D, Aron L, O'Hern P, Dhamne SC, Davidsohn N, Mao CA, Klein WH, Rotenberg A, Bennett DA, Church GM, Colaiácovo MP, Yankner BA (2019) Regulation of lifespan by neural excitation and REST. Nature 574:359-364. 